\title{
On the cortical mapping function - visual space, cortical space, and crowding
}

\author{
Hans Strasburger \\ University of München, University of Göttingen \\ $\underline{\text { www.hans.strasburger.de }}$
}

For submission to Journal of Vision

\begin{abstract}
The retino-cortical visual pathway is retinotopically organized: Neighborhood relationships on the retina are preserved in the mapping to the cortex. Size relationships in that mapping are also highly regular: The size of a patch in the visual field that maps onto a cortical patch of fixed size, follows, along any radius and in a wide range, simply a linear function with retinal eccentricity. This is referred to as $M$-scaling. As a consequence, and under simplifying assumptions, the mapping of retinal to cortical location follows a logarithmic function along a radius, as was already shown by Fischer (1973) and Schwartz $(1977,1980)$. The M-scaling function has been determined for many visual tasks. It is standardly characterized by its foveal threshold value, together with the eccentricity where that value doubles, called $E_{2}$. The cortical location function, on the other hand, is commonly specified by parameters that are separately determined from the empirical findings. Here, the psychophysical and neuroscience traditions are brought together by specifying the cortical equations in terms of the parameters customary in psychophysics. The equations go beyond those published in the past in being more explicit and ready for application, and they allow easy switching between $M$-scaling and cortical mapping. A new parameter, $d_{2}$, is proposed to describe the cortical map, as a cortical counterpart to $E_{2}$ and typical values for it are given. The resulting cortical-location function is then applied to data from a number of fMRI studies. One pitfall is discussed and spelt out as a set of equations, namely the common myth that a pure logarithmic function will give an adequate map: The popular omission of a constant term renders the equations ill-defined in and around the retinotopic center. The correct equations are finally extended to describe the cortical map of Bouma's law on visual crowding. The result contradicts recent suggestions that critical crowding distance corresponds to constant cortical distance.
\end{abstract}

Keywords: Cortical map, logarithmic map, cortical magnification, visual cortex, $M$-scaling, $E_{2}$ value, retinotopy, Bouma's Law, crowding, myths, visual field

\section{Introduction}

One of the most beautiful organizational principles of the human brain is that of topographical mapping. Whilst perhaps universal to the brain, its regularity is most apparent for the three primary senses mediated through the thalamus - sight, hearing, and touch i.e., in retinotopy, tonotopy, and somatotopy. For the visual domain with which we are concerned here, the regularity of topography is particularly striking and is at a level that lends itself to mathematical description by analytic functions. The seminal papers by Fischer 
(1973) and Schwartz $(1977,1980)$ derive the complex logarithm as a suitable function for mapping the location in the visual field to the location of its projection's in (a flat-map of) the primary visual cortex, by which the visual field's polar-coordinate grid gets mapped onto a rectilinear cortical grid. The log function's image domain - the complex plane - is reinterpreted thereby as a two-dimensional real plane. ${ }^{1}$ As Schwartz explains in the two papers ${ }^{2}$, the rationale for employing the log function in the radial direction is that its first derivative is an inverse linear function, as implicit in the cortical magnification concept for the visual field as proposed by Daniel \& Whitteridge (1961) (see the next section for explanations of the concepts, or Strasburger, Rentschler, \& Jüttner, 2011, Section 3, for review). Expressed more directly, the integral of an inverse linear function (as implied in the cortical magnification concept) is the logarithmic function. Intuitively, summing-up (integrating over) little steps on the cortical map where each step obeys cortical magnification will result in the log mapping.

Schwartz's $(1977,1980)$ papers with the complex-log mapping have become rather popular in visual psychophysics and visual neurophysiology ${ }^{3}$. Van Essen, Newsome \& Maunsell (1984), e.g., use it for explaining the topography of the macaque's primary visual cortex and write, "Along the axis corresponding to constant polar angle, magnification is inversely proportional to eccentricity, and hence distance is proportional to the logarithm of eccentricity $(x \propto \log E) "$ (p. 437). Klein \& Levi (1987) derive, from the log rule that, if vernieracuity offsets are assumed to have a constant cortical representation (i.e. one that is independent of eccentricity), vernier offsets will depend linearly on eccentricity in the visual field. Horton \& Hoyt (1991) use it to point out that the well-known inverse linear function for cortical magnification follows from a log-spaced cortical map. Engel et al. (1997, Fig. 9, Fig 12) and Larsson \& Heeger (2006) use the (real-valued) log function implicitly when they use an exponential for the inverse location function (which corresponds to a log forward mapping). Duncan \& Boynton (2003) fit their fMRI activity maps for the V1 topology using Schwartz's complex-log mapping. Providing an easy-to-apply closed-form mathematical basis for these mappings with explicit parameter equations by deriving what I will call the cortical location function will be the first major goal of the present paper.

While Fischer's and Schwartz's papers present the mathematical relationships and give examples for their application, Klein \& Levi (1987) go further and provide an empirical link between basic psychophysical data in the visual field and the cortical map. For characterizing the psychophysical results they use a concept they had developed earlier (Levi, Klein, \& Aitsebaomo, 1984; Levi, Klein, \& Aitsebaomo, 1985): The slope of the normalized thresholdsvs.-eccentricity function can be quantified by a single number, called $E_{2}$. In an x-y plot of, say, the vernier threshold shown across eccentricity, that value is the (negative) $X$-axis intercept, or, alternatively, the (positive) eccentricity value at which the foveal threshold value doubles

\footnotetext{
${ }^{1}$ Note that the elegance of the complex-log representation is deceiving in that not all properties of the complex plane have a counterpart in the $2 \mathrm{D}$ real plane (which is undesirable for a mathematical representation). For example, the square of a value on the upper vertical meridian does not correspond to a value on the left horizontal meridian, as would be implied by $i^{2}=-1$.

2 "The fact that the radial magnitude of the cortical magnification factor can be approximated by an inverse linear function implies that the mapping function for the cortex might be the complex logarithm, because the derivative of this function does indeed have an inverse linear form ... However, other mapping functions [also] have the same radial logarithmic structure." (Schwartz, 1980, p. 647)

3 ... with 348 and 385 citations as of Aug. 2019. Fischer's (1973) paper, even though it contains the original derivations, received fewer (101) citations.
} 
(illustrated below in Figure 1B). To draw the link to the cortical logarithmic map, Klein \& Levi (1987) show that relationships become simpler and more accurate, if both psychophysical and cortical data are not treated as a function of eccentricity $(E)$ itself but of a transformed eccentricity, $E^{*}$, referred to as effective eccentricity. $E^{*}$ is defined as $E^{*}=E+E_{2}$. In the visual field, the linear cortical magnification function thereby turns into a simple proportionality. In the cortical map, distances are then proportional to the logarithm of effective eccentricity, $\mathrm{x} \propto \log \left(E+E_{2}\right)$. The approach is verified by showing the empirical data both as thresholds and in cortical units (Klein \& Levi, 1987, Fig. 5) ${ }^{4}$.

However, the papers discussed so far have not yet fully exploited the tight mathematical link between psychophysics and the cortical map for its empirical use. The basic mathematical form of the mapping function - $\log (E)$ or $\log \left(E+E_{2}\right)$ - is drawn upon and made use of but further parameters are usually left free to vary and to be determined by data fitting. The derivations in the present paper thus take the log mapping approach one step further. Unlike in Schwartz (1980), Klein \& Levi (1987), and quite a few other papers discussed below, the parameters for the logarithmic map are here obtained by mathematical derivation from those in the visual field. For the latter, i.e., for the psychophysical characterization, Levi and Klein's $E_{2}$ concept is again the basis. We thereby arrive at a set of fully explicit equations that allow converting the psychophysical description by $E_{2}$ to a description in the cortical map. These equations are the message of the paper. The empirical data for the cortical maps (from $\mathrm{fMRI}$ or single-cell analysis) are then, in a next step, used to verify the correctness of those parametrical equations. This approach represents a more principled one. It further places additional constraints on the describing functions, thus adding to their reliability.

Since such derivations have been attempted before and have led to erroneous results (e.g. in our own writing), or stopped short of exploring the implications, care is taken here to present the derivations step-by-step, considering at each step what that means. Key result equations are highlighted by surrounding boxes for easy spotting, i.e. those that should be of practical use in describing the cortical map, or, e.g., for obtaining improved estimates for the foveal cortical magnification factor.

Instead of the complex log, we here consider the simpler case of real-valued, 1D mapping, where eccentricity in the visual field, expressed in degrees of visual angle along a radius, is mapped onto the distance of its representation from the retinotopic center, expressed in millimeters. The resulting real-valued logarithmic function shall be called the cortical location function. This implies no loss of generality; that function is easily generalized to the $2 \mathrm{D}$ case by writing it as a vector function. Compared to the complex log, the real function has the added advantage of allowing separate parameters for the horizontal and vertical meridian, required to meet the horizontal-vertical anisotropy of the visual field. ${ }^{5}$

Once these relationships for the cortical location function are established, they need to be verified by empirical data. We use data from the literature and own data for this. It turns out that not only do the fits work excellently, but the constraints imposed by the parametric equations can be used for the long-standing problem of improving estimates for the foveal cortical magnification factor $\left(M_{0}\right)$. In one section, it is further argued that the simplified

\footnotetext{
${ }^{4}$ For rescaling the right ordinate in that figure, they used the relationship that $1 \mathrm{~mm}$ of cortex corresponds to approximately $10 \%$ of the effective eccentricity.

${ }^{5}$ A closed-form analytic representation using the complex log function, yet allowing for the horizontal-vertical anisotropy, is presented by Schira, Tyler, Spehar \& Breakspear (2010).
} 
version $(x \propto \log E)$ that is occasionally used in the $\mathrm{fMRI}$ literature needs to be avoided and the full version with a constant term added in the log's argument $(x \propto \log (E+c))$ needs to be employed.

Finally, the cortical location function can be used (perhaps unexpectedly) to derive the cortical distances in visual crowding. Crowding happens when neighboring patterns to a target stimulus are closer than a critical distance, where the latter can be described by Bouma's law (Bouma, 1970; Pelli \& Tillman, 2008; Strasburger, 2020). We thus arrive at a cortical version of Bouma's law. While this has been done before (Levi et al., 1985; Motter \& Simoni, 2007; Pelli, 2008; Nandy \& Tjan, 2012; Strasburger et al., 2011; Strasburger \& Malania, 2013), the present derivations go beyond Levi et al. (1985), Motter \& Simoni (2007), Pelli (2008), and Nandy \& Tjan (2012) in that they include the fovea, and go beyond Strasburger et al. (2011) and Strasburger \& Malania (2013) in that they provide the derivations and avoid derivation errors.

\section{Goals of the Paper}

Three goals are thus pursued in this paper. Firstly, relationships are derived that translate the nomenclature of psychophysics to that in cortical physiology. The approaches are closely linked, and results on the mapping functions can be translated back and forth. The key equations for the cortical location function will be eq. (10) and eq. (16) plus (17). The usefulness of these equations is shown in a subsequent section.

Secondly, it is explored how the cortical function looks like in the popular simplified case with omitted constant term (Figure 2 below). That section can be skipped if one is aware that this seemingly simpler method will lead to suboptimal and misleading results. It is argued there that this might have been a good solution at a time but is not now when we have detailed knowledge of the cortical mapping close to the retinotopic center. The linear $M$-scaling function shown below in Figure 1 is accurate down to very low eccentricities, which is not the case for the simplified location function. It makes little sense to continue working with equations that do not, and cannot, apply over the whole range.

Conversely, the usefulness of the derived improved equations is shown in a section that explores practical examples for the cortical mapping function, with data from the literature. The graphs look like those in Figure 2 but have realistic parameter values. Comprehensive and concise mathematical descriptions have been derived before (Schira, Tyler, et al., 2010); the purpose here is to do so with explicit cortical parameters using the nomenclature from psychophysics, (i.e., using $E_{2}$ ), in an easily applicable way.

Thirdly, and finally, these concepts are applied to the cortical map for visual crowding. Crowding, i.e. the impaired recognition of a pattern in the presence of neighboring patterns, is probably the prominent characteristic of peripheral vision. Remarkably, unlike typical perceptual tasks like acuity where critical size scales with eccentricity, crowding is mostly independent of target size. Instead of size, the critical distance between target and flankers scales with eccentricity. This characteristic has become to be known as Bouma's law. It follows the same linear eccentricity law as depicted in Figure 1 below; this time, however, it refers to distance between patterns instead of size of patterns. Consequently, the $E_{2}$ concept can be applied in the same way. The cortical location function derived in the first part can then be used to predict the cortical distances that correspond to the flanker distances in Bouma's law. 
Guide to reading the paper. The key parts of the paper are Section 2 on the cortical location function and Section 3 on its extension to Bouma's law in the cortex. These are preceded by Section 1 on the involved concepts and history, which can be skipped if one is already familiar with that background.

Regarding the structuring of Section 2, it starts with the natural way of specifying the cortical location function which is relative to the retinotopic center (Section 2.1). However, that center is notoriously difficult to locate exactly, and so for the neuroscientist it is of interest to be able to use some other reference location instead. Ways to do that, and their limitations, are discussed in Section 2.2 and 2.3. The latter of these (2.3), however, is intended to show how not to do it and can be skipped if that is agreed upon. Next is a section on practical examples (2.4), with three cases from the literature, further a method for estimating the foveal magnification factor $\left(M_{0}\right)$, and finally the introduction of a new metric $\left(d_{2}\right)$ for characterizing the cortical map that is the equivalent of $E_{2}$ in the cortex.

Section 3 for Bouma's law in the cortex can be read mostly independently of the preceding sections if the underlying location function from Section 2 is taken for granted.

In the derivations, care was taken to phrase the steps to be easy to follow. Yet a legitimate way of using the paper is to just take the highlighted final results as take-home message. These would be eq. (9), (10) or (13) for the cortical mapping of the visual field (i.e. the cortical location function), eq. (8a) for the new parameter $d_{2}$, eq. (17) for $M_{0}$, and eq. (32) (34), or (38) - (40), for the mapping of Bouma's rule onto the cortex.

\section{Background and concepts}

Peripheral vision is unlike central vision as Ptolemy (90-168) already noted. Ibn al-Haytham (965-1040) was the first to study it quantitatively. Purkinje (1787-1869) determined the dimensions of the visual field with his sophisticated perimeter. Aubert and Foerster (1857) started modern quantitative research on the gradual variation across visual field eccentricities. Østerberg (1935) did meticulous measurements of retinal rod and cone receptor densities across the horizontal meridian (Strasburger et al, 2011, Fig. 4); they are still a part of modern textbooks on perception (see Wade, 1998, Strasburger \& Wade, 2015a, 2015b, and Strasburger et al., 2011, for review).

Yet we still lack a grip on what the nature of peripheral vision is. The goal here in the paper is to draw the attention to the highly systematic organization of neural input stage, by deriving equations that describe its retino-cortical architecture. But before we delve into the nittygritty of the equations in the main part, some background and the concepts involved are reviewed, to see the equations in perspective.

Peripheral vs. central vision: qualitative or quantitative difference? Whether the difference between central and peripheral vision is of a qualitative or a quStrasburger et al., 2011antitative nature has long been, and still is, an issue of debate. Early perceptual scientists suggested a qualitative (along with a quantitative) difference: Al-Haytham, in the $11^{\text {th }}$ century, wrote that "form [...] becomes more confused and obscure" in the periphery (Strasburger \& Wade, 2015a). Porterfield (1696-1771) pointed out the obscurity of peripheral vision and called its apparent clearness a "vulgar error". Jurin's (1738, p. 150) 
observation that complexity of objects plays a role ${ }^{6}$ suggests that more than a simple quantitative change is at play (and reminds of crowding). Similarly, Aubert \& Foerster (1857, p. 30) describe the peripheral percept of several dots as "something black of indetermined form". Yet, perhaps due to the lack of alternative concepts (like Gestalt perception) or by a prevailing interest in the role of vision in astronomy, the underlying reasons for the differences were then invariably ascribed to a purely quantitative change of a basic property: spatial resolution. Trevarthen's (1968) two-process theory of focal, detail-oriented central vision, vs. ambient, space-oriented peripheral vision, might seem a prominent example of a qualitative distinction. However, with its emphasis on separate higher cortical areas for the two roles (perhaps nowadays dorsal vs. ventral processing), it does not speak to qualitative differences in the visual field's low-level representation.

On the quantitative side, concepts for the variations across the visual field only emerged in the $19^{\text {th }}$ century. Aubert and Foerster's (1857) characterization of the performance decline with retinal eccentricity as a linear increase of minimum resolvable size - sometimes referred to as the Aubert-Foerster Law - is still the conceptual standard. It corresponds to what is now called M-scaling (Virsu \& Rovamo, 1979; Virsu, Näsänen, \& Osmoviita, 1987) or the change of local spatial scale (Watson, 1987). However, by the end of the $19^{\text {th }}$ century it became popular to use the inverse of minimum resolvable size instead, i.e. acuity, in an attempt to make the decline more graphic (e.g. Fick, 1898). And, since the inverse of a linear function's graph is close to a hyperbola, we arrive at the well-known hyperbola-like function of acuity vs. eccentricity seen in most textbooks, or in Østerberg's (1935) figure from which they are derived.

The hyperbola graph. Graphic as it may be, the familiar hyperbola graph implicit, e.g., in $\emptyset$ sterberg's (1935) receptor-density graph does not lend itself easily to a comparison of decline parameters. Weymouth (1958) therefore argued for returning to the original use of a non-inverted size by introducing the concept of the minimal angle of resolution (MAR). Not only as an acuity measure but also as a generalized size threshold. Based on published data, Weymouth summarized how the MAR and other spatial visual performance parameters depend on retinal eccentricity (MAR, vernier threshold, motion threshold in dark and light, Panum-area diameter and others, see Weymouth, 1958, e.g. Fig. 13). Importantly, Weymouth stressed the necessity of a non-zero, positive axis intercept for these functions. ${ }^{7}$ This will be a major point here in the paper; it is related to the necessity of a constant term in the cortical-location function discussed below. The architecture of neural circuitry in the visual field thus appears to be such that processing units increase in size and distance from each other towards the periphery in retinal space. To Weymouth, these processing units were the span of connected receptor cells to individual retinal ganglion cells. Different

\footnotetext{
6 "when we divide [a string of digits] so as to constitute several objects less compounded, we can more easily estimate the number of figures" (Jurin, 1738, p. 150). Jurin reports more examples that would count as qualitative differences; see Strasburger \& Wade (2015a).

7 "If the threshold as a function of eccentricity were a straight line passing through the origin (this does not occur and would require an infinite foveal sensitivity) the threshold would be a constant percentage of the eccentricity. It is here claimed that these curves approximate a straight line, but with a finite and positive intercept; this would lead to a decreasing percentage, falling, at first, rapidly but changing more and more slowly in the periphery. The 'constant' percentage relation noted by Ogle is therefore a consequence of the straight line relationship here discussed and is secondary and less useful mathematically. Although Ogle must have observed this linear relationship, he does not seem to have developed its consequences as is here done." (Weymouth, 1958, p. 109); italics added.
} 
slopes, Weymouth (1958) suggested, might arise from differing task difficulty, a view not shared by later authors, however.

Cortical magnification. The linear spatial concept was thus well established when in the sixties and seventies the cortex was taken into the picture and the role of cortical representation included in theories on visual field inhomogeneity. Daniel \& Whitteridge (1961) and Cowey \& Rolls (1974) introduced cortical magnification as a unifying concept which, for a given visual-field location, summarizes functional density along the retinocortical pathway into a single number, $M$. Linear $M$ was defined as the diameter in the primary visual cortex onto which 1 deg of the visual field projects (alternatively, areal $M$ was defined as the cortical area onto which $1 \mathrm{deg}^{2}$ projects). Enlarging peripherally presented stimuli by $M$ was shown to counter visual-performance decline to a large degree for many visual tasks (reviewed, e.g., by Virsu et al., 1987) and was thus suggested as a general means of equalizing visual performance across the visual field (Rovamo \& Virsu, 1979). Yet this socalled strong hypothesis was soon dismissed; an early critique was expressed by Westheimer (1982) on the grounds that vernier acuity thresholds cannot be explained with these concepts. $^{8}$

Even though the relationship between the early visual architecture and psychophysical tasks is still a matter of debate and, with it, the question why different visual tasks show widely differing slopes of their eccentricity functions (see Figure 1), the variation of the cortical magnification factor with eccentricity is largely agreed upon: $M$ decreases with eccentricity following approximately an hyperbola - and its inverse, $M^{-1}$, increases linearly. Klein \& Levi (1987) point out that by replacing eccentricity by effective eccentricity $E^{*}=E+E_{2}$, the dependency turns into proportionality (i.e., twice $E^{*}$ leads to twice $M^{-1}$ ). The value of $M$, and its variation with eccentricity, can be determined anatomically or physiologically (Schwartz, 1980; Van Essen et al., 1984; Tolhurst \& Ling, 1988; Horton \& Hoyt, 1991, Slotnick, Klein, Carney, \& Sutter, 2001, Duncan \& Boynton, 2003; Larsson \& Heeger, 2006; Schira, Wade, \& Tyler, 2007; see Figure 1, reproduced from Fig. 9 in Strasburger et al., 2011). Assuming that low-level tasks like measuring the MAR reflect cortical scaling, $M$ can also be estimated psychophysically (Rovamo \& Virsu, 1979; Virsu \& Rovamo, 1979; Virsu et al., 1987).

\footnotetext{
8 "Psychophysical procedures do not, therefore, provide a single unambiguous measure for the changes of spatial grain across the visual field." (Westheimer, 1982, p. 157). And later: "There is a rather insistent opinion abroad that spatial visual processing has identical properties right across the visual field save for a multiplicative factor which is a function of eccentricity." (p. 161). The term "spatial grain" in the paper's title refers to cortical units. With respect to an explanation for vernier acuity, Westheimer writes "If the actual threshold value is a manifestation of a complex cortical processing apparatus, the distance over which it operates optimally is the more likely parameter to be found correlated with the anatomical representation of the visual field in the cortex, and this, for some reason, does not show the gross increase in grain exhibited by the threshold data." (p. 162)
} 

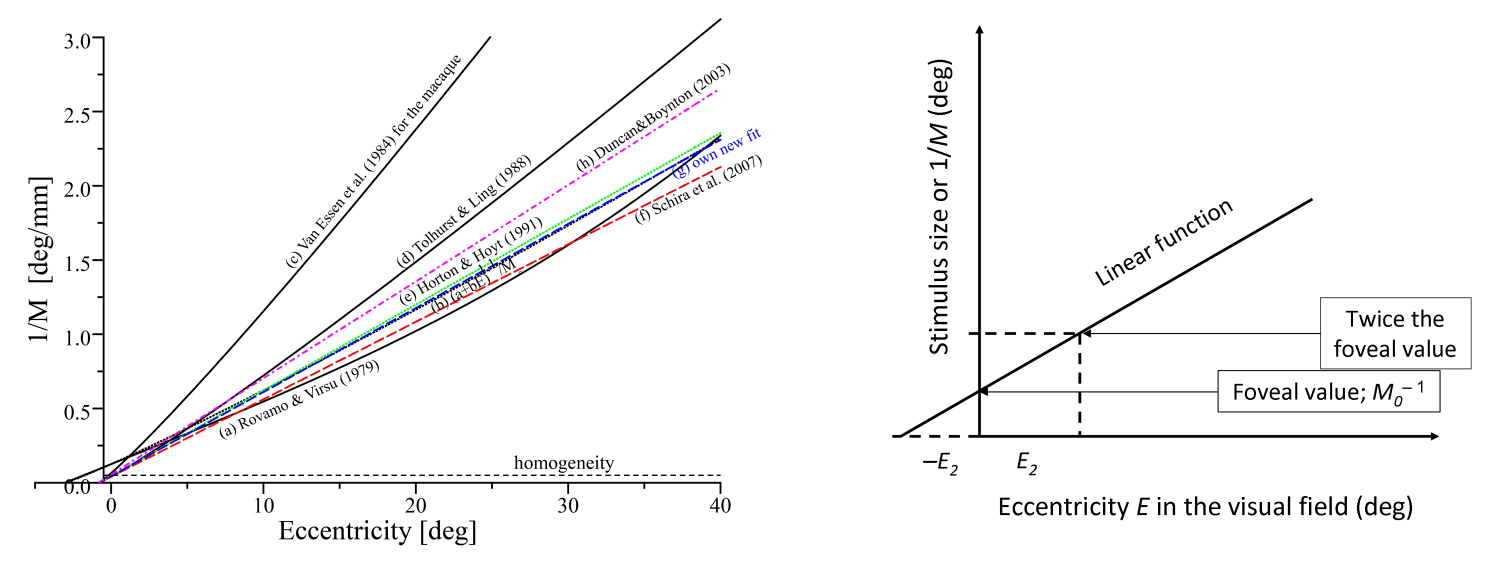

Figure 1. A. The inverse of the cortical magnification factor, or - equivalently - the size of a patch in the visual field that projects onto a patch of constant size in the cortex, as a function of eccentricity in the visual field (Fig. 9 in Strasburger et al., 2011, reproduced for illustrating the text). All functions show a mostly linear behavior. Their slope is quite similar, with the exception of Van Essen et al.'s (1984) data for the macaque; other data show similar slopes between human and monkey (e.g. Oehler, 1985). B. An illustration of the $E_{2}$ concept.

The empirical data all fit the linear concept quite well, but some slight deviations are apparent in the considered range of about $40^{\circ}$ eccentricity. These are asides here but should be mentioned. The linear equation for the eccentricity function was often "tweaked" a little to accommodate for these deviations: Rovamo, Virsu, \& Näsänen (1978) added a small $3^{\text {rd }}$ order term, Van Essen et al. (1984) and Tolhurst \& Ling (1988) increased the exponent of the linear term slightly, from 1 to 1.1. Virsu \& Hari (1996) took a different approach and used a sine function, based on geometrical considerations. Only a part of the sine's period was used (one-eighth) though, so that the function is still close to linear in that range. The latter function is interesting because it is the only one that - because it is bounded - can be extended to larger eccentricities, $90^{\circ}$ and even beyond that (note that the visual field extends beyond $90^{\circ}$; Strasburger, 2020).

Elliptical field. Another deviation from simple uniform linearity is the fact that the visual field is not isotropic: Performance declines differently between radii (this is used by Greenwood, Danter, \& Finnie, 2017, to disentangle retinal from cortical distance). Iso-performance lines for the binocular field are approximately elliptical rather than circular outside the central visual field (e.g. Wertheim, 1894, Harvey \& Pöppel, 1972; Pöppel \& Harvey, 1973, see their Fig. 6). At the transition from the isotropic to the anisotropic field (in the plateau region of Pöppel \& Harvey, 1973), the scaling functions (Figure 1A) not only have different slopes along the different meridians but also necessarily deviate from linearity. Correspondingly, early visual areas are also anisotropic (e.g. Horton \& Hoyt, 1991). The effect of anisotropy on the cortical magnification factor is quantitatively treated by Schira et al. $(2007,2010)$; their $M_{0}$ estimate is the geometric mean of the isopolar and isoeccentric $M$ estimates. In the equations presented below, the anisotropy can be accommodated by letting the parameters depend on the radius in question. However, different parameters (slopes) along the radii in the log mapping are not sufficient to adequately account for the anisotropy, as Schira et al. $(2007,2010)$ have shown. For preserving area constancy across meridians, these authors thus extend the model by a shear function (using the hyperbolic secans; Schira et al., 2010, 
eq. 6 and Fig. 2) such that mappings differ between meridians, with deviations from linearity on the vertical meridian, and meridians close to that, at around $1^{\circ}$ eccentricity (see Schira et al., 2010, Fig. 2). The derivations presented here, for simplicity, do not include these refinements and are thus only approximate for the vertical meridian.

The $E_{2}$ concept. For a quick comparison of eccentricity functions for psychophysical tasks, Levi et al. (1984, p. 794) introduced $E_{2}-$ a value which denotes the eccentricity at which the foveal threshold for the corresponding task doubles (Figure 1B illustrates this). More generally, $E_{2}$ is the eccentricity increment at which the threshold increases by the foveal value. As a graphic aide, note that this value is also the distance from the origin of where the linear function crosses the eccentricity axis (i.e., $E_{2}$ is the negative abscissa intercept in Figure 1B).

Eq. (1) below states the equation using the $E_{2}$ parameter. The function's slope is given by the fraction $M_{0}^{-1} / E_{2}$, so when these functions are normalized to the foveal value their slope is $E_{2}^{-1} . E_{2}$ thus captures an important property of the functions in a single number. A summary of values was reported, e.g., by Levi et al. (1984, Levi et al., 1985), Klein \& Levi (Klein \& Levi, 1987), or more recently by Strasburger et al. (2011, Tables $4-6)$. These reported $E_{2}$ values vary widely between different visual functions. They also vary considerably for functions that seem directly comparable to each other (Vernier: $0.62-0.8 ; M^{-1}$ estimate: $0.77-0.82$;

Landolt-C: 1.0-2.6; letter acuity: 2.3-3.3; gratings: $2.5-3.0$ ). Note also the limitations of $E_{2}$ : since the empirical functions are never fully linear for example, the characterization by $E_{2}$, by its definition, works best at small eccentricities.

The two centers. There is an important difference in difficulty between assessing the fovea's center and the cortical retinotopic center. Whereas, for psychophysical tests, the measurement of the foveal value is particularly simple and reliable, the opposite is the case for the anatomical foveal counterpart, $M_{0}{ }^{-1}$. The latter is considered the most difficult to determine and is mostly extrapolated from peripheral values. The consequences of this include a different perspective on research on the map between the two fields. We will come back to that below.

Using the $E_{2}$ parameter, the inverse-linear scaling function can be concisely and elegantly stated as

$M^{-1} / M_{0}^{-1}=1+E / E_{2}$.

$M^{-1}$ in that equation, measured in $\% \mathrm{~mm}$, is the inverse cortical magnification factor as defined above; $M_{0}^{-1}$ is that value in the fovea's center. The left hand ratio in the equation, $\mathrm{M}^{-1} / \mathrm{M}_{0}^{-1}$, is the ratio by which a peripherally seen stimulus needs to be size-scaled to occupy cortical space equal to a foveal stimulus. So the equation can equally well be written as

$S / S_{0}=1+E / E_{2}$

where $S$ is scaled size and $S_{0}$ is the size at the fovea's center. From eq. (1), $M_{0}{ }^{-1}$ can be considered the size-scaling unit in the visual field, and $E_{2}$ the locational scaling unit (i.e. the unit in which scaled eccentricity is measured).

Cortical mapping: As mentioned above, Fischer $(1973)$ and Schwartz $(1977,1980)$ proposed the complex log function for mapping the visual field to the cortical area. The key property of interest for that mapping, however, is the behavior along a radius from the fovea in the 
visual field, which corresponds to the simpler real-valued log function. This then maps the eccentricity in the visual field to the distance from the retinotopic center on the cortical map. Neuroscience papers often prefer to show the inverse function (i.e. mirrored along the diagonal with the $x$ and $y$ axis interchanged, thus going "backwards" from cortical distance to eccentricity), which is the exponential function shown schematically in Figure 2. Schwartz (1980) has discussed two versions of the function that differ in whether there is a constant term added in the argument; the difference is illustrated in the graph. The version without the constant is often considered simpler and is thus often preferred (or the full version is ignored). An important point in the following will be that that simplicity is deceiving and can lead to wrong conclusions (and more complicated equations). The proposed term location function can refer to both the forward ( $\log$ ) and backward (exp) version, which are synonymous.

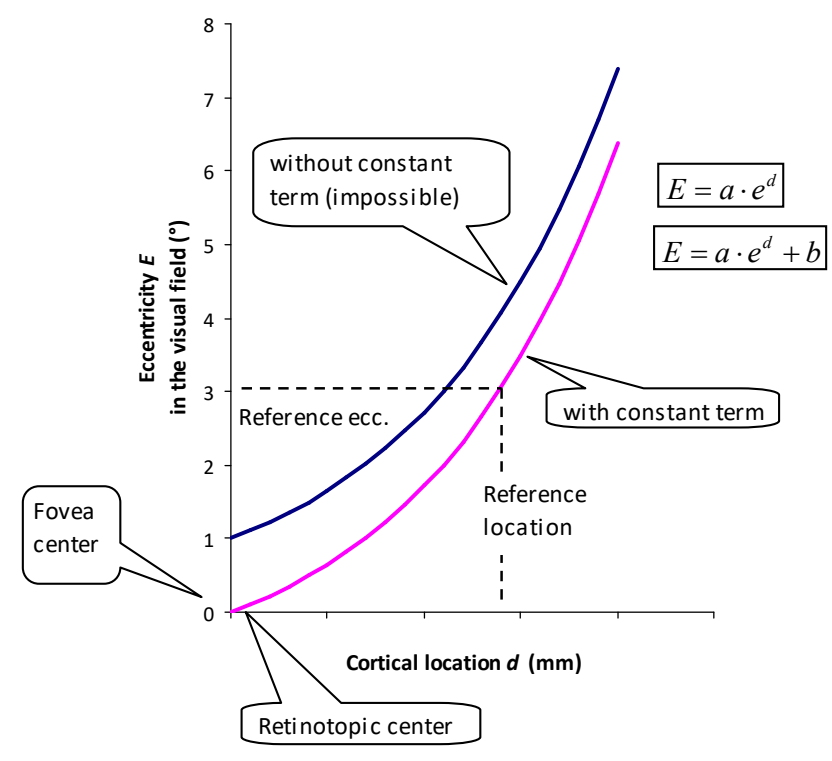

Figure 2. Illustration of the cortical location function introduced by Fischer (1973) and Schwartz (1977, 1980). A version with, and another without a constant term (parameter $b$ in the equation) is shown. The constant term's omission was intended as a simplification for large eccentricities but is physically impossible for the foveal center. The graph shows $E$ as a function of $d$, which is an exponential; Schwartz (1980) discussed mainly the inverse function, i.e. for cortical distance $d$ as a function of eccentricity $E$, which is logarithmic.

Symbols in the paper: To keep a better overview, symbols used in the paper are summarized in Table 1. Some of those are in standard use; some are newly introduced in the remainder. 


\begin{tabular}{|l|l|l|}
\hline & Visual Field & Cortical Map \\
\hline Cortical magnification factor & $M^{-1}$ & $M$ \\
\hline Stimulus size & $S$ & - \\
\hline Location as distance from the center & $E$ & $d$ \\
\hline Location as distance from a reference & - & $d$ \\
\hline Levi and Klein's $E_{2}$ & $E_{2}$ & $d_{2}$ \\
\hline Location of reference as distance from the center & - & $d_{\text {ref }}$ \\
\hline Critical distance for crowding & $\delta$ & $\kappa$ \\
\hline Critical distance for crowding in the very center & $\delta_{0}$ & $\kappa_{0}$ \\
\hline$E_{2}$ for critical crowding distance & $\hat{E}_{2}$ & - \\
\hline
\end{tabular}

Table 1. Summary of symbols used in the paper

\section{The cortical location function}

\subsection{Cortical location specified relative to the retinotopic center}

The ratio $\mathrm{M}^{-1} / \mathrm{Mo}_{0}^{-1}$ in eq. (1) is readily estimated in psychophysical experiments as the size of a stimulus relative to a foveal counterpart for achieving equal perceptual performance in a low-level task. However, in physiological experiments $M$ is difficult to assess directly, even though it is a physiological concept. Instead, it is typically derived - indirectly - from the cortical-location function $d=d(E)$ (Figure 2). The function links a cortical distance $d$ in a retinotopic area to the corresponding distance in the visual field that it represents. More specifically, $d$ is the distance (in $\mathrm{mm}$ ) on the cortical surface between the representation of a visual-field point at eccentricity $E$, and the representation of the fovea center. Under the assumption of linearity of the cortical magnification function $M^{-1}(E)$, this function is logarithmic (and its inverse $E=E(d)$ is exponential as in Figure 2), as shown by Fischer (1973) and Schwartz $(1977,1980)$. And since $E_{2}$ allows a simple formulation of cortical magnification function in psychophysics, as e.g. in eq. 1 , it will be useful to state the equation $d=d(E)$ with those notations. This is the first goal of the paper. The location function allows a concise quantitative characterization of the early retinotopic maps (symbols used in the paper are summarized in Table 1).

To derive the cortical location function, notice first that, locally, the cortical distance of the respective representations $d(E)$ and $d(E+\Delta E)$ of two nearby points along a radius at eccentricities $E$ and $E+\Delta E$ is given by $M(E) \cdot \Delta E$. This follows from $M$ 's definition (and that $M$ refers to $\left.1^{\circ}\right)$. The cortical magnification factor $M$ is thus the first derivative of $d(E)$,

$M=d^{\prime}(E)$.

Conversely, the location $d$ on the cortical surface is the integral over $M$ (starting at the fovea center):

$d(E)=\int_{0}^{E} M(E) d E$.

If we insert eq. (1) (i.e. the equation favored in psychophysics) into eq. (4), we have 


$$
\begin{aligned}
d(E) & =\int_{0}^{E} \frac{M_{0}}{1+E / E_{2}} d E \\
& \left.=M_{0} E_{2} \ln \left(1+E / E_{2}\right) \text { (with } E \geq 0\right),
\end{aligned}
$$

where $\ln$ denotes the natural logarithm (cf. Schwartz, 1980).

The inverse function, $E(d)$, which is derived by inverting eq. (5), is

$E=E_{2}\left(e^{\frac{d}{M_{0} E_{2}}}-1\right)$ (with $\left.d \geq 0\right)$.

It states how the eccentricity $E$ in the visual field depends on the distance $d$ of the corresponding location in a retinotopic area from the point representing the fovea center. With slight variations (discussed below) it is the formulation often referenced in fMRI papers on the cortical mapping. Note that, by its nature, it is only meaningful for positive values of cortical distance $d$.

We can simplify that function further, by introducing an analogue to $E_{2}$ in the cortex. Like any point in the visual field, $E_{2}$ has a representation (i.e. on the meridian in question) and we denote the distance $d$ of its location from the retinotopic center as $d_{2}$. Thus, $d_{2}$ in the cortex represents $E_{2}$ in the visual field.

To express eq. (6) using $d_{2}$ instead of $M_{0}$, first apply the equation to that location $d_{2}$ :

$E_{2}=E_{2}\left(e^{\frac{d_{2}}{M_{0} E_{2}}}-1\right)$.

Solving this for the product $M_{0} E_{2}$,

$M_{0} E_{2}=d_{2} / \ln 2$,

and inserting that into eq. (6) gives

$$
E=E_{2}\left(2^{d / d_{2}}-1\right)
$$

Eq. (9) is the most concise way of stating the cortical location function. However, since the exponential to the base e is often more convenient, we restate it as

$$
E=E_{2}\left(e^{(\ln 2) d / d_{2}}-1\right)
$$

(here, In again denotes the natural logarithm).

This equation (eq. 10) is particularly nice and simple provided that $d_{2}$, the cortical equivalent of $E_{2}$, is known. That value, $d_{2}$, could thus play a key role in characterizing the cortical map, similar to the role of $E_{2}$ in visual psychophysics (cf. Table 4 - Table 6 in Strasburger et al., 2011, or earlier the tables in Levi et al., 1984, Levi et al., 1985, or Klein \& Levi, 1987). Estimates for $d_{2}$ derived from literature data are summarized in Section 2.4 below, as an aid for concisely formulating the cortical location function.

The new cortical parameter $d_{2}$ can be calculated from eq. (8), restated here for convenience:

$$
d_{2}=M_{0} E_{2} \ln 2
$$




\subsection{Cortical location specified relative to a reference location}

Implicit in the definition of $d$ or $d_{2}$ is the knowledge about the location of the fovea center's cortical representation, i.e. of the retinotopic center. However, that locus has proven to be hard to determine precisely. Instead of the center it has thus become customary to use some fixed eccentricity $E_{\text {ref }}$ as a reference. Engel et al. (1997, Fig. 9), for example, use $E_{\text {ref }}$ $=10^{\circ}$. Larsson \& Heeger (2006, Fig. 5) use $E_{\text {ref }}=3^{\circ}$.

To restate eq. (6) or (10) accordingly, i.e. with some reference eccentricity different from $E_{\text {ref }}=0$, we first apply eq. (10) to that reference:

$E_{r e f}=E_{2}\left(e^{(\ln 2) d_{r e f} / d_{2}}-1\right)$,

where $d_{\text {ref }}$ denotes the value of $d$ at the chosen reference eccentricity, e.g. at $3^{\circ}$ or $10^{\circ}$.

Solving that equation for $d_{2}$ and plugging the result into eq. (9) or (10), we arrive at

$E=E_{2}\left(\left(E_{r e f} / E_{2}+1\right)^{d / d_{r e f}}-1\right)$.

Expressed to the base e, we have

$$
E=E_{2}\left(e^{\beta\left(d / d_{r e f}\right)}-1\right) \text {, with } \beta=\ln \left(\frac{E_{r e f}}{E_{2}}+1\right)(\text { and } d \geq 0),
$$

which represents the location function expressed relative to a reference eccentricity $E_{\text {ref }}$ and its equivalent in the cortical map, $d_{\text {ref }}$ (one could also derive eq. (13) directly from eq. (6)). Note that if, in that equation, $E_{2}$ is taken as the reference eccentricity for checking, it reduces to eq. (10) as expected. So, $E_{2}$ can be considered as a special case of a reference eccentricity. Note further that, unlike the location equations often used in the retinotopy literature (Van Essen et al., 1984, in the introduction; Duncan \& Boynton, 2003; Larsson \& Heeger, 2006), the equations are well defined in the fovea center: for $d=0$, eccentricity $E$ is zero, as it should be.

What reference to choose is up to the experimenter. However, the fovea center itself cannot be used as a reference eccentricity - the equation is undefined for $d_{r e f}=0$ (since the exponent is then infinite). The desired independence of knowing the retinotopic center's location has thus not been achieved: That knowledge is still needed, since $d$, and $d_{\text {ref, }}$ in these equations are defined as the respective distances from that point.

Equations (12) and (13) have the ratio $d / d_{\text {ref }}$ in the exponent. It is a proportionality factor for $d$ from the zero point. From the intercept theorem we know that this factor cannot be reexpressed by any other expression that leaves the zero point undefined. True independence from knowing the retinotopic center, though desirable, thus cannot be achieved.

We can nevertheless shift the coordinate system such that locations are specified relative to the reference location, $d_{\text {ref. }}$. For this, we define a new variable $\hat{d}$ as the cortical distance (in $\mathrm{mm}$ ) from the reference $d_{\text {ref }}$ instead of from the retinotopic center (see Figure 3 for an illustration for the shift and the involved parameters), where $d_{\text {ref }}$ is the location corresponding to some eccentricity, $E_{\text {ref. }}$ By definition, then,

$\hat{d}=d-d_{r e f}$ 


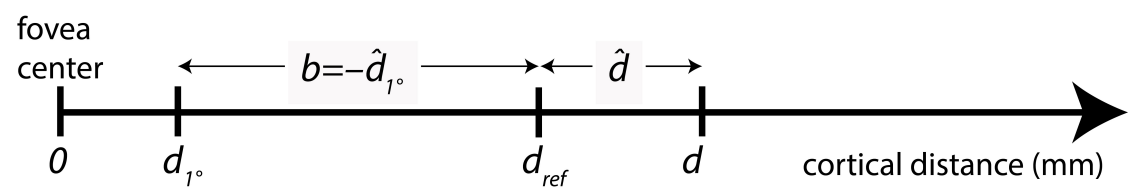

Figure 3: Illustration of the cortical distance measures used in equations (6) - (23), and of parameter $b$ in eq. (18).

$d$ - cortical distance of some location from the retinotopic center, in $\mathrm{mm}$;

$d_{\text {ref }}$ - distance (from the center) of the reference that corresponds to $E_{\text {ref; }}$;

$d_{1}$ 。 distance of the location that corresponds to $E=1^{\circ}$;

$\hat{d}$ - distance of location $d$ from the reference $d_{\text {ref. }}$.

In the shifted system - i.e., with $\hat{d}$ instead of $d$ as the independent variable - eq. (6) for example becomes

$$
E=E_{2}\left(e^{\frac{\hat{d}+d_{r e f}}{M_{0} E_{2}}}-1\right) \text {. }
$$

The equation might be of limited practical use, however, like eq. (6) from which it was derived, since the parameters $M_{0}$ and $E_{2}$ in it are not independent; they are inversely related to each other as seen in eq. (8) or (8a) (or eq. 17). That interdependency is removed in eq. (9) or (10), (which work from the retinotopic center), or eq. (13) (which used a reference eccentricity). The latter (eq. 13), in the shifted system becomes

$$
E=E_{2}\left(e^{\beta \frac{\hat{d}+d_{r e f}}{d_{r e f}}}-1\right) \text {, with } \beta=\ln \left(\frac{E_{r e f}}{E_{2}}+1\right)\left(\text { and } \hat{d}+d_{r e f} \geq 0\right) \text {. }
$$

That equation now has the advantage over eq. (15) of having only two free parameters, $E_{2}$ and $d_{r e f}\left(E_{r e f}\right.$ is not truly free since it is empirically linked to $\left.d_{\text {ref }}\right)$. The foveal magnification factor $M_{0}$ has dropped from the equation. Indeed, by comparing eq. (13) to eq. (6) (or by comparing eq. (15) to (16)), $M_{0}$ can be calculated from $d_{r e f}$ and $E_{2}$ as

$$
M_{0}=\frac{d_{r e f}}{\beta \cdot E_{2}}
$$

where $\beta$ is defined as in the previous equation. With an approximate location of the retinotopic center (needed for calculating $d_{\text {ref }}$ ) and an estimate of $E_{2}$, that latter equation leads to an estimate of the foveal magnification factor, $M_{0}$ (see Section 2.4 for examples).

Equations (16) and (17) are crucial to determining the retinotopic map in early areas. They should work well for areas V1 to V4 as discussed below. The connection between the psychophysical and physiological/fMRI approaches in these equations allows cross-validating the empirically found parameters and thus leads to more reliable results. Duncan \& Boynton (2003), for example, review the linear law and also determine the cortical location function empirically but do not draw the connection. Their's and others' approaches are discussed as practical examples in the section after next (Section 2.4). 


\subsection{Independence from the retinotopic center with the simplified function?}

Schwartz (1980) had offered a simplified location function (where the constant term is omitted) which works at sufficiently large eccentricities. Frequently that was the preferred one in later papers as being (seemingly) being more practical. The present section will show how this approach will lead astray if pursued rigorously; it can be skipped, i.e. is not required for following the subsequent sections.

The simplified version of the location function $E(\hat{d})$ omits the constant term in eq. (6) and those that follow from it (i.e., the " -1 " in eq. 6 to eq. 16). I.e., the equation

$$
E=e^{a(\hat{d}+b)}
$$

is fit to the empirical data, with free parameters $a$ and $b$. The distance variable in it is then $\hat{d}$ as before, i.e., the cortical distance in $\mathrm{mm}$ from a reference that represents some eccentricity $E_{\text {ref }}$ in the visual field. Engel et al. (1997, Fig. 9), for example, use $E_{r e f}=10^{\circ}$ for such a reference, and for that condition the reported equation is $E=\exp (0.063(\hat{d}+36.54))$. Larsson \& Heeger (2006, Fig. 5), as another example, use $E_{r e f}=3^{\circ}$, and for area V1 in that figure give the function $E=\exp (0.0577(\hat{d}+18.0))$.

For a better understanding of these equations we can attach meaning to the parameters $a$ and $b$ in eq. (18) by looking at two special points, $\hat{d}=0$ and $\hat{d}=-\mathrm{b}$ : At the point $\hat{d}=-b$, eccentricity $E$ equals $1^{\circ}$ visual angle (from the equation), so we call that value $\hat{d}_{1^{\circ}}$. For parameter $b$ we thus have

$b=-\hat{d}_{1}$ 。

The value of $\hat{d}_{1}$ is negative; it is around $-36.5 \mathrm{~mm}$ for $E_{\text {ref }}=10^{\circ}$ and is the distance of the $1^{\circ}$ line from the reference eccentricity's representation (where $\hat{d}=0$ ). At $\hat{d}=0$, on the other hand, we have $E=E_{\text {ref }}$ by definition, and from eq. (18) it follows that

$a=\left(\ln E_{r e f}\right) / b=-\left(\ln E_{r e f}\right) / \hat{d}_{1^{\circ}}$.

Now that we have parameters $a$ and $b$ we can insert those in the above equation and rearrange terms, by which we get

$E=E_{r e f} \cdot E_{r e f}^{-\hat{d} / \hat{d}_{1^{\circ}}}$

or, expressed more conveniently to the base e,

$E=E_{r e f} \cdot e^{\left(\ln E_{r e f}\right)\left(-\hat{d} / \hat{d}^{\circ}\right)}$.

This is now the simplified cortical location function (the simplified analog to eq. 16), with parameters spelt out. One can easily verify that the equation holds true at the two defining points, i.e. at $1^{\circ}$ and at the reference eccentricity. Note also that, as intended, knowing the retinotopic center's location in the cortex is not required since $\hat{d}$ is defined relative to a nonzero reference. Obviously, however, the equation fails increasingly with smaller eccentricities, for the simple reason that $E$ cannot become zero. In other words, the fovea's center is never reached, even (paradoxically) when we are at the retinotopic center. Equation (18), or (21), (22) are thus better avoided.

To observe what goes wrong towards eccentricities closer to the fovea center, let us express the equation relative to the absolute center. From eq. (14) and 
$\hat{d}_{1^{\circ}}=d_{1^{\circ}}-d_{r e f}$

it follows

$E=E_{r e f} \cdot e^{\left(\ln E_{r e f}\right) \frac{d-d_{r e f}}{d_{r e f}-d_{1}^{\circ}}}$,

where $d$, as before, is the distance from the retinotopic center. Naturally, by its definition, the equation behaves well at the two defining points (resulting in the values $E_{r e f}$, and $1^{\circ}$, respectively). However, in between these two points the function has the wrong curvature (see Fig. 4 in the next section), and at the fovea center (i.e., at $d=0$ ), the predicted eccentricity - instead of zero - takes on a meaningless non-zero value $E_{0}$ given by

$$
E_{0}=E_{r e f} \cdot e^{\left(\ln E_{r e f}\right) \frac{d_{r e f}}{d_{1}-d_{r e f}}} .
$$

As seen in the equation, the value depends on the chosen reference eccentricity and its representation, and the cortical representation of $1^{\circ}$ eccentricity, all of which should not happen. So, the seeming simplicity of eq. (18) that we started out from leads astray in and around the fovea (which, after all, is of prime importance for vision). The next section illustrates the differences between the two sets of equations with data from the literature.

\subsection{Practical use of the equations: examples}

\subsubsection{The approach of Larsson \& Heeger (2006)}

Now that we have derived two sets of equations for the location function (i.e. with and without a constant term in Section 2.1 and 2.3, respectively) let us illustrate the difference with data on the cortical map. The first example are data from Larsson \& Heeger (2006, Fig. 5) for V1. As a reminder, this is about eq. (16) on the one hand - in essence $E=a\left(e^{b \hat{d}}-1\right)$, derived from eq. (6) - and the discouraged eq. (24) on the other hand ( $E=a e^{b \hat{d}}$, derived from eq. (18).

For the reasons explained above the retinotopic center is left undefined by Larsson \& Heeger (2006) and a reference eccentricity of $E_{r e f}=3^{\circ}$ is used instead. The fitted equation in the original graph is stated as $E=\exp (0.0577(\hat{d}+18.0))$, which corresponds to eq. (18) with constants $a=0.0577$ and $b=-\hat{d}_{1^{\circ}}=18.0$. Its graph is shown in Figure 4 as the thick black line copied from the original graph. It is continued to the left as a dotted blue line to show the behavior toward the retinotopic center. At the value of $-b$, i.e. at a distance of $\hat{d}_{1^{\circ}}=-18.0$ $\mathrm{mm}$ from the $3^{\circ}$ representation (as seen from eq. 19), the line crosses the $1^{\circ}$ point. To the left of that point, i.e. towards the retinotopic center, the curve deviates markedly upward and so the retinotopic center $\left(E=0^{\circ}\right)$ is never reached. 


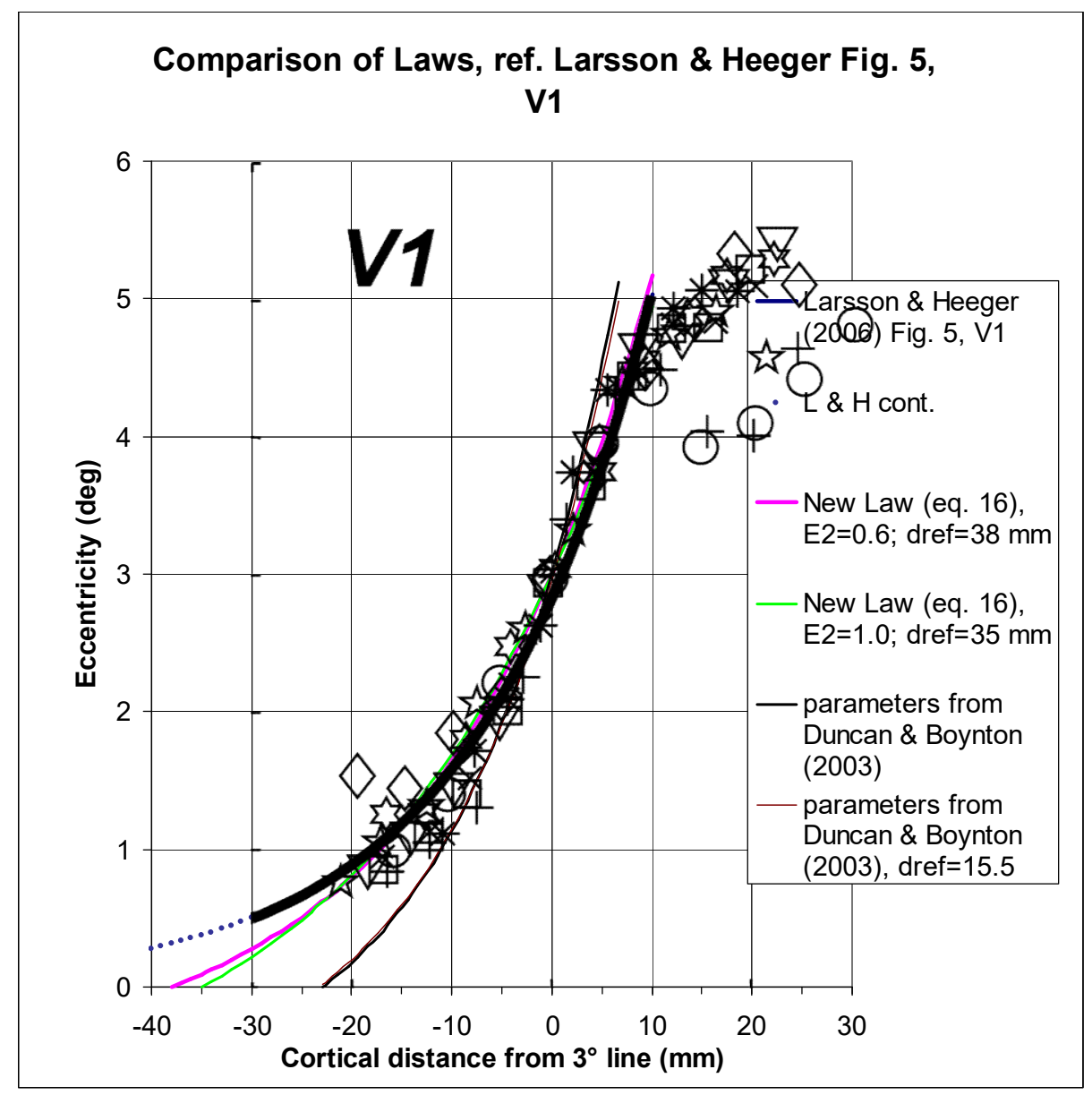

Figure 4. Comparison of conventional and improved analytic functions for describing the cortical location function (retinal eccentricity vs. corresponding cortical location). Symbols show the retinotopic data for area V1 with $d_{r e f}=3^{\circ}$ from Larsson and Heeger (2006, Fig. 5) (symbols for nine subjects), together with the original fit (fat black line), according to eq. (18) $(E=\exp (a(\hat{d}+b)))$ or (22), i.e. a fit without a constant term). The blue dotted line continues that fit to lower eccentricities; the fitted $E(\hat{d})$ function goes to (negative) infinite cortical distance, which is physically meaningless. Pink and green line: graphs of the preferable eq. (16), derived from integrating the inverse linear law (eq. 1), with two different parameter choices; $\left[E_{2}=0.6^{\circ}, d_{\text {ref }}=38 \mathrm{~mm}\right]$ and $\left[E_{2}=1.0^{\circ}, d_{\text {ref }}=35 \mathrm{~mm}\right]$, respectively. The retinotopic center's magnification factor $M_{0}$ can be calculated by eq. (17) as $35.4 \mathrm{~mm} /{ }^{\circ}$ and $25.3 \mathrm{~mm} /{ }^{\circ}$ for the two cases, respectively. Black and brown line: $E(\hat{d})$ function with parameters derived by Duncan \& Boynton (2003), $M_{0}=18.5 \mathrm{~mm} /{ }^{\circ}$ and $E_{2}=0.831^{\circ}$ (black), and with $d_{\text {ref }}=15.5 \mathrm{~mm}$ (brown) for comparison (discussed in the next section). Note that, by definition, the curves from Larsson \& Heeger pass through the $3^{\circ}$ point at $\hat{d}=0 \mathrm{~mm}$. Note also that data beyond $\sim 10 \mathrm{~mm}$ were said to be biased by the authors and can be disregarded.

The pink and the green curve in Figure 4 are two examples for a fit of the equation with a constant term (i.e., for eq. 16). The pink curve uses $E_{2}=0.6^{\circ}$ and $d_{\text {ref }}=38 \mathrm{~mm}$, and the green curve $E_{2}=1.0^{\circ}$ and $d_{\text {ref }}=35 \mathrm{~mm}$. Note that smaller $E_{2}$ values go together with larger $d_{\text {ref }}$ values for a similar shape. Within the range of the data set, the two curves fit about equally well; the pink curve is slightly more curved (a smaller $E_{2}$ is accompanied by more curvature). Below about $1^{\circ}$ eccentricity, i.e. around half way between the $3^{\circ}$ point and the retinotopic center, the two curves deviate markedly from the original fit. They fit the data better there 
and, in particular, they reach a retinotopic center. The pink curve (with $E_{2}=0.6^{\circ}$ ) reaches the center at $38 \mathrm{~mm}$ from the $3^{\circ}$ point, and the green curve at $35 \mathrm{~mm}$.

The center cortical magnification factor, $M_{0}$, for the two curves can be derived from eq. (17), giving a value of $35.4 \mathrm{~mm} /{ }^{\circ}$ and $25.3 \mathrm{~mm} /{ }^{\circ}$, respectively. These two estimates differ substantially - by a factor of 1.4 - even though there is only a 3-mm difference of the assumed location of the retinotopic center. This illustrates the large effect of the estimate for the center's location on the foveal magnification factor, $M_{0}$. It also illustrates the importance of a good estimate for that location.

There is a graphic interpretation of the foveal magnification factor $M_{0}$ in these graphs. From eq. (6) one can derive that $M_{0}^{-1}$ is equal to the function's slope at the retinotopic center. Thus, if the function starts more steeply (as does the green curve compared to the pink one), $M_{0}{ }^{-1}$ is higher and thus $M_{0}$ is smaller.

The figure also shows two additional curves (black and brown), depicting data from Duncan \& Boynton (2003), which are discussed below. To better display the various curves' shapes, they are shown again in Figure 5 but without the data symbols. Figure 5 also includes an additional graph, depicting the exponential function $E=\exp (0.063(\hat{d}+36.54))$ reported by Engel et al. (1997). In it, $\hat{d}$ is again the cortical distance in millimeters but this time from the $10^{\circ}$ representation. $E$, as before, is the visual field eccentricity in degrees. For comparison with the other curves, the curve is shifted (by $19.1 \mathrm{~mm}$ ) on the abscissa to show the distance from the $3^{\circ}$ point. The curve runs closely with that of Larsson \& Heeger (2006) and shares its difficulties.

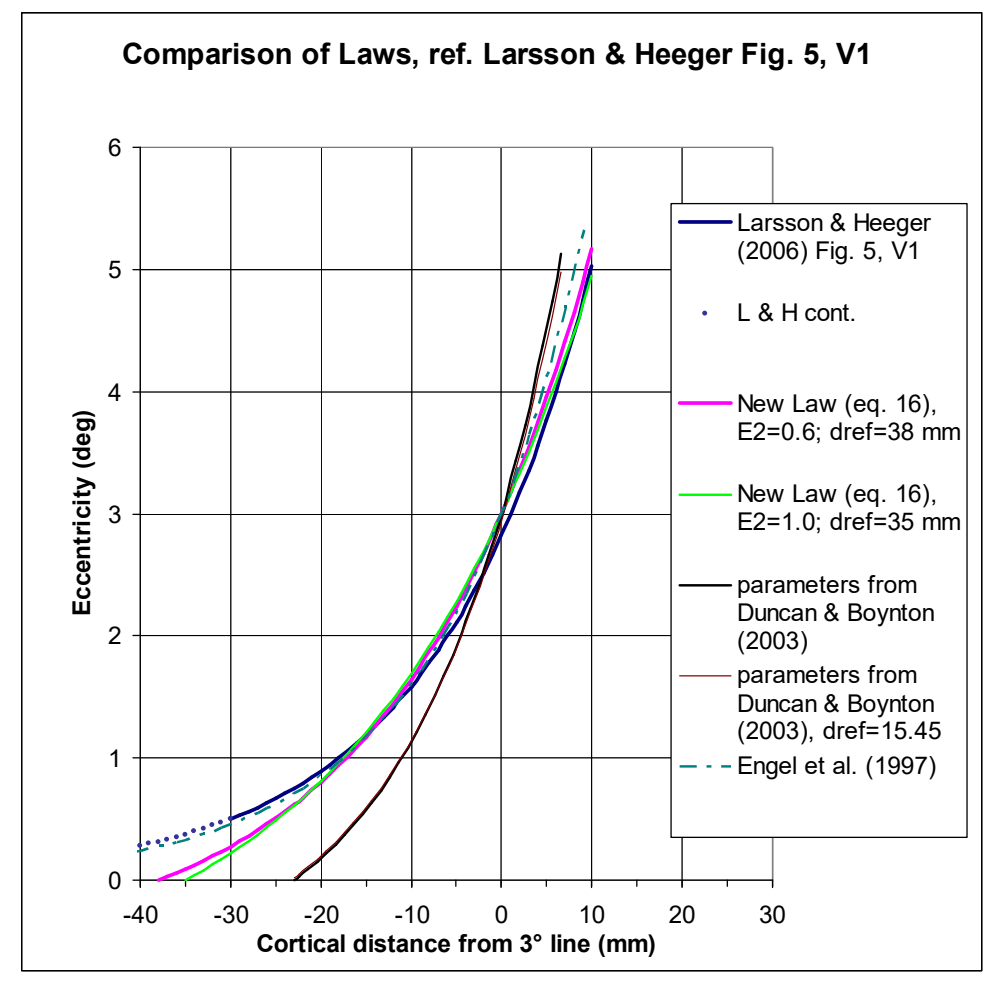


Figure 5. Same as Figure 4 but without the data symbols, for better visibility of the curves. The additional dash-dotted curve next to that of Larsson \& Heeger's depicts the earlier equation by Engel et al. (1997).

\subsubsection{The approach of Duncan \& Boynton (2003)}

In addition to the curves just discussed, Figure 4 and Figure 5 show a further $E(\hat{d})$ function that is based on the results of Duncan \& Boynton (2003). That function obviously differs quite a bit from the others in the figure and it is thus worthwhile studying how Duncan \& Boynton (2003) derived these values. The paper takes a somewhat different approach for estimating the retinotopic mapping parameters for $\mathrm{V} 1$ than the one discussed before.

As a first step in Duncan \& Boynton's paper, the locations of the lines of equal eccentricity are estimated for five eccentricities $\left(1.5^{\circ}, 3^{\circ}, 6^{\circ}, 9^{\circ}, 12^{\circ}\right)$ in the central visual field, using the equation $w=k^{*} \log (z+a)$. The function looks similar to the ones discussed above, except that $z$ is now a complex variable that mimics the visual field in the complex plane. On the horizontal half-meridian that is equivalent to eq. (6) in the present paper, i.e., to an $E(d)$ function that includes a constant term (parameter $a$ ) and with the retinotopic center as the reference. At these locations, the authors then estimate the size of the projection of several $1^{\circ}$-patches of visual space (see their Fig. 3 ; this is where they differ in their methodology). By definition, these sizes are the cortical magnification factors $M_{i}$ at the corresponding locations. Numerically, these sizes are then plotted vs. eccentricity in the paper's Fig. 4. Note, however, that this is not readily apparent from the paper, since both the graph and the accompanying figure caption state something different. In particular the $y$-axis is reported incorrectly (as is evident from the accompanying text). For clarity, therefore, Figure 6 here plots these data with a corrected label and on a linear $y$-axis.
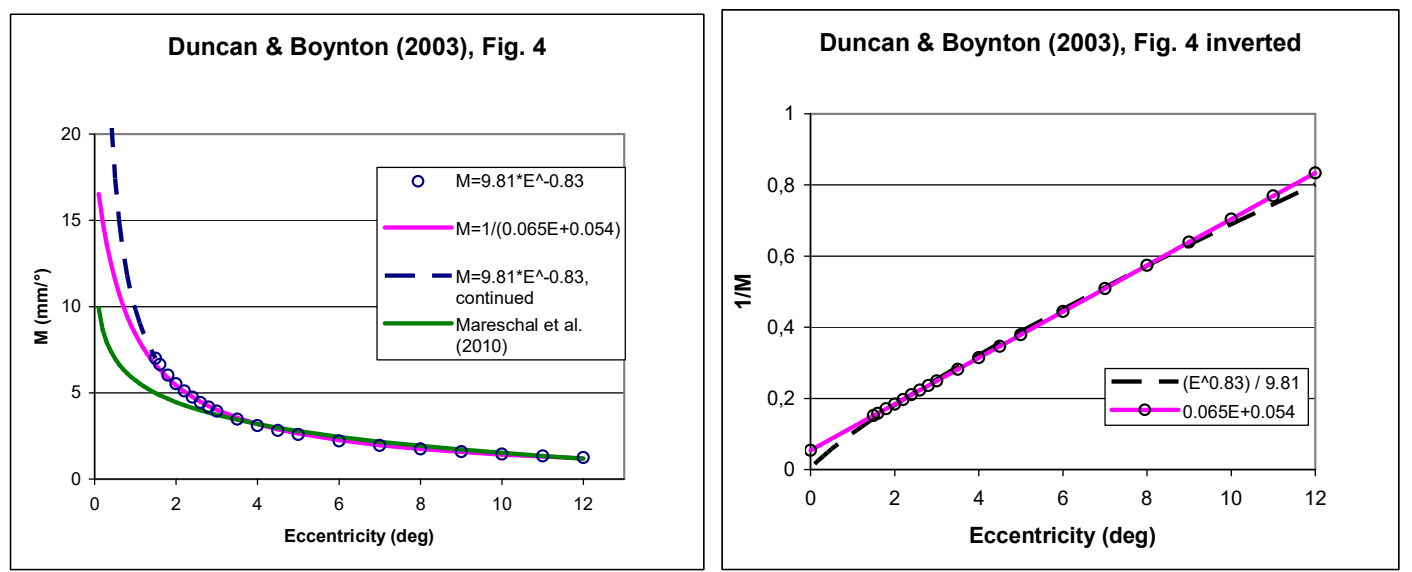
Figure 6. (A) Duncan \& Boynton's (2003) Fig. 4, showing the cortical magnification factor's variation with eccentricity drawn on a linear $y$-axis and with a corrected $y$-axis label $\left(M\right.$ in $\left.\mathrm{mm} /{ }^{\circ}\right)$. Note that the equation proposed earlier in the paper (p. 662), $M=9.81^{*} E^{-0.83}$, predicts an infinite foveal magnification factor (blue curve). In contrast, the inverse-linear fit $M^{-1}=0.065 E+0.054$ proposed later in the paper (p. 666) fits the data equally well in the measured range of $1.5^{\circ}$ to $12^{\circ}$ but predicts a reasonable foveal magnification factor of $18.5 \mathrm{~mm} /{ }^{\circ}$. The $E_{2}$ value for the latter equation is $E_{2}=0.83$. The additional green curve shows an equation by Mareschal et al. (2010) (see next section). (B) The inverse of the same functions. Note the slight but important difference at $0^{\circ}$ eccentricity, where the linear function is non-zero and its inverse is thus welldefined.

The authors next fit a power function to those data, stated as $M=9.81^{*} E^{-0.83}$ for the cortical magnification factor (see Figure 6). There is a little more confusion, however, because it is said that from such power functions the foveal value can be derived by extrapolating the fit to the fovea (p. 666). That cannot be the case, however, since - by the definition of a power function (including those used in the paper) - there is no constant term. The function therefore goes to infinity towards the fovea center, as shown in Figure 6 (dashed line). Furthermore, $E_{2}$, which is said to be derived in this way in the paper, cannot be derived from a nonlinear function (because the $E_{2}$ concept requires a linear or inverse-linear function). The puzzle is resolved with a reanalysis of Duncan \& Boynton's Fig. 4. It reveals how the foveal value and the connected parameter $E_{2}$ were, in fact, derived: as an inverse-linear function which fits the data equally well in the measured range of $1.5^{\circ}-12^{\circ}$ eccentricity (Figure 6, continuous line). From that function, the foveal value and $E_{2}$ are readily derived. Indeed, the two values correspond to the values given in the paper.

The distance of the isoeccentricity lines from the retinotopic center is not specified in Duncan \& Boynton (2003). We can derive that from eq. (17), though, because $M_{0}$ and $E_{2}$ are fixed:

$d_{\text {ref }}=M_{0} \beta E_{2}$.

With their parameters $\left(M_{0}=18.5 \mathrm{~mm} /{ }^{\circ}\right.$ and $\left.E_{2}=0.83\right)$, the scaling factor $\beta$ in that equation comes out as $\beta=1.03$ (from eq. 16). From that, $d_{\text {ref }}=d_{1.5^{\circ}}=15.87 \mathrm{~mm}$. As a further check we can also derive a direct estimate of $d_{\text {ref }}$ from their Fig. 3 . For subject ROD, for example, the $1.5^{\circ}$ line is at a distance of $d_{1.5^{\circ}}=15.45 \mathrm{~mm}$ on the horizontal meridian. That value is only very slightly smaller than the one derived above. For illustration, Figure 4 and Figure 5 in the previous section also contain a graph for that value (thin black line). Conversely, with $d_{\text {ref }}$ given, $M_{0}$ can be derived from eq. (17) (or eq. 26), which gives a slightly smaller value of $M_{0}$ $=18.0 \mathrm{~mm} /{ }^{\circ}$. The two curves are hardly distinguishable; thus, as previously stated, $d_{\text {ref }}$ and $M_{0}$ interact, with different value-pairs resulting in similarly good fits.

In summary, the parameters in Duncan \& Boynton's (2003) paper: $M_{0}=18.5 \mathrm{~mm} /{ }^{\circ}$ and $E_{2}=0.83$, are supported by direct estimates of the size of $1^{\circ}$-projections. They are taken at locations estimated from a set of mapping templates, which themselves are derived from a realistic distance-vs.-eccentricity equation. The paper provides another good example how the linear concept for the magnification function can be brought together with the exponential (or logarithmic) location function. The estimate of $M_{0}$ comes out considerably lower than in more recent papers (e.g. Schira et al., 2009; see Figure 7 below). Possibly the direct estimation of $M$ at small eccentricities is less reliable than the approach taken in those papers. 


\subsubsection{Mareschal, Morgan \& Solomon (2010)}

Figure 6 shows an additional curve from a paper by Mareschal et al. (2010) on cortical distance, who base their cortical location function partly on the equation of Duncan \& Boynton (2003). Mareschal et al. (2010) state their location function as

$$
M^{\prime}(E)= \begin{cases}(0.065 E+0.054)^{-1} & E<4^{\circ} \\ 5.72-\log _{1.73}(E) & E>4^{\circ}\end{cases}
$$

The upper part of the equation is that of Duncan \& Boynton (pink curve) and is used below an eccentricity of $4^{\circ}$. The green continuous line shows Mareschal's log equation above $4^{\circ}$, and the dashed line shows how the log function would continue for values below $4^{\circ}$. Obviously, the latter is not meaningful and is undefined at zero eccentricity, which is why Mareschal et al. then switched to the inverse-linear function (i.e. the pink curve). The problem at low eccentricity is apparent in Fig. 9 in their paper where the $x$-axis stops at $1 / 2$ deg, so the anomaly is not fully seen. For their analysis the switch of functions is not relevant since eccentricities other than $4^{\circ}$ and $10^{\circ}$ were not tested. The example is just added here as an illustration that the new equations derived here would have allowed for a single equation, with no need for case distinctions.

\subsubsection{Toward the retinotopic center}

As discussed above, predictions of the retinotopic center depend critically on its precise location and thus require data at small eccentricities. Schira, Tyler and coworkers have addressed that problem in a series of papers (Schira et al., 2007; Schira, Tyler, Breakspear, \& Spehar, 2009; Schira et al., 2010) and provide detailed maps of the centers of the early visual areas, down to $0.075^{\circ}$ eccentricity. They also develop parametric, closed analytical equations for the $2 \mathrm{D}$ maps. When considered for the horizontal ${ }^{9}$ direction only, these equations correspond to those discussed above (eq. 1 and eq. 16/17) ${ }^{10}$.

Figure 7 shows magnification factors from Schira et al., 2009, Fig. 7A, with figure part B showing their V1 data (red curve), redrawn on double-linear coordinates. As can be seen, the curve runs close to an hyperbola. Its inverse is shown in Figure 7C, which displays the familiar, close-to-linear behavior over a wide range, with a positive $y$-axis intercept that corresponds to the value at the fovea center, $M_{0}^{-1}$. From the regression line, $M_{0}$ and $E_{2}$ are readily obtained and are $E_{2}=0.21^{\circ}$ and $M_{0}=47.6 \mathrm{~mm}$, respectively. Note that a rather large value of $M_{0}$ is obtained compared to previous reports. However, as can also be seen from the graph, if one disregards the most peripheral point, the centrally located values predict a somewhat shallower slope of the linear function with a thus slightly larger $E_{2}$ and smaller $M_{0}$ value: $E_{2}=0.33^{\circ}$ and $M_{0}=34.8 \mathrm{~mm}$. The latter values might be the more accurate predictors for V1's central point.

\footnotetext{
${ }^{9}$ The equations differ on radians close to, and on, the vertical meridian since Schira and Tyler have introduced a shear factor for preserving area constancy across meridians (Schira et al., 2007 Schira et al., 2010).

${ }^{10}$ The equations presented here go further in that they draw the direct connection to the $E_{2}$ nomenclature, i.e. that they provide a link to psychophysics.
} 

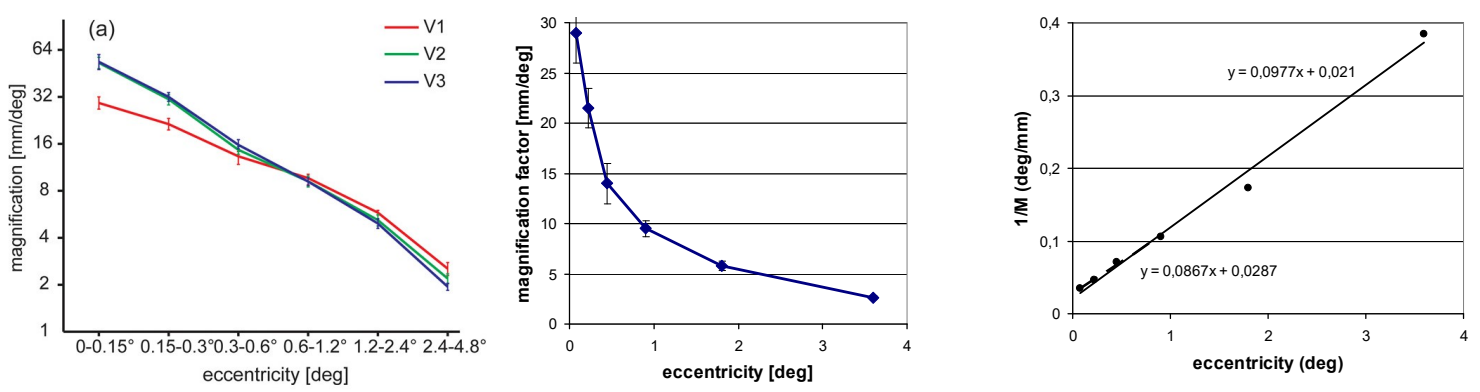

Figure 7. Cortical magnification factor from Schira, Tyler, Breakspear \& Spehar (2009, Fig. 7A). (A) Original graph. (B) V1 data for $M$ from Schira et al.'s graph but drawn on double-linear coordinates. (C) Resulting inverse factor, again on linear coordinates. The regression line, $M^{-1}=0.0977 E+0.021$, fits the whole set and predicts $E_{2}=0.21^{\circ}$ and $M_{0}=47.6 \mathrm{~mm}$. The regression equation $M^{-1}=0.0867 E+0.0287$ is a fit to the first four points and might be a better predictor for the retinotopic center, giving the values $E_{2}=0.33^{\circ}$ and $M_{0}=34.8 \mathrm{~mm}$.

In summary, the derived equations provide a direct link between the nomenclature used in psychophysics and that in neurophysiology on retinotopy. They were applied to data for $\mathrm{V} 1$ (Fig. 2) but will work equally well for higher early visual areas, including V2, V3, and V4 (cf. Larsson \& Heeger, 2006, Fig. 5; Schira et al., 2009, Fig. 7). Mo is expected to be slightly different for the other areas (Schira et al., 2009, Fig. 7)) and so will likely be the other parameters.

\subsection{5 $d_{\mathbf{2}}$ - a parameter to describe the cortical map}

As shown in Section 2.1 (eq. 9 or 10), a newly defined parameter $d_{2}$ can be used to describe the cortical location function very concisely. Parameter $d_{2}$ is the cortical representation of Levi and Klein's $E_{2}$, i.e. the distance (in $\mathrm{mm}$ from the retinotopic center) of the eccentricity, $E_{2}$, where the foveal value doubles. Eq. (8) can serve as a means to obtain an estimate for $d_{2}$. Essentially, $d_{2}$ is the product of $M_{0}$ and $E_{2}$ with a scaling factor. Table 2 gives a summary of $d_{2}$ estimates thus derived. 


\begin{tabular}{|c|c|c|c|c|}
\hline Study & $M_{0}[\mathrm{~mm}]$ & $E_{2}\left[^{\circ}\right]$ & $d_{2}[\mathrm{~mm}]$ & Curve \\
\hline Larsson \& Heeger (2006) & 35.4 & 0.6 & 14.72 & Fig. 4, pink \\
\hline “ & 25.3 & 1.0 & 17.54 & Fig. 4, green \\
\hline Duncan \& Boynton (2003) & 18.5 & 0.831 & 10.66 & Fig. 4, black \\
\hline Schira, Tyler, Breakspear \& Spehar (2009) & 47.6 & 0.21 & 6.93 & Fig. 7C \\
\hline " & 34.8 & 0.33 & 7.96 & Fig. $7 C, 2^{\text {nd }}$ regression \\
\hline D'Souza, Auer, Frahm, Strasburger \& Lee (2016, Fig. 4) & $32.32 *$ & 0.45 & 10.08 & |L-M| Channel \\
\hline " & $32.32 *$ & 0.97 & 21.73 & Lum Channel \\
\hline “ & $32.32 *$ & 3.4 & 76.17 & S Channel \\
\hline
\end{tabular}

Table 2. Values of the parameter $d_{2}$ from a reanalysis of data in several studies, by eq. (8): $d_{2}=M_{0} E_{2} \ln (2)$. $d_{2}$ is the cortical representation of $E_{2}$ and characterizes the cortical location function in a single value.

${ }^{*} M_{0}$ was not estimated in that paper; the mean of the preceding $M_{0}$ values was used for the calculation instead.

\section{Crowding and Bouma's Law in the cortex}

The preceding sections were about the cortical location function; in the final section the derived location function will be applied to an important property of cortical organization: visual crowding. Thus, whereas in the preceding, cortical location was the target of interest, in this section we are concerned with cortical distances.

As reviewed in the introduction, MAR-like functions like acuity generally change in peripheral vision in that critical size scales with eccentricity, so deficits can (mostly) be compensated for by $M$-scaling (as, e.g. in Rovamo \& Virsu, 1979). For crowding, in contrast, target size plays little role (Strasburger, Harvey, \& Rentschler, 1991; Pelli, Palomares, \& Majaj, 2004). Instead, the critical distance between target and flankers scales with eccentricity, though at a different rate than MAR. This scaling characteristic of crowding is known as Bouma's rule or Bouma's law (Bouma, 1970; Strasburger et al., 1991; Pelli et al., 2004; Pelli \& Tillman, 2008; Strasburger, 2020). The corresponding distances in the primary cortical map are thus governed by differences of the cortical location function as derived here in Section 2. Crowding's critical distance (or indeed any distance, including acuity gap size) is thus, in a sense, a spatial derivative of location. Pattern recognition, at even slight eccentricities is, governed by the crowding phenomenon and is largely unrelated to visual acuity (or thus to cortical magnification) (Strasburger et al., 1991; Pelli et al., 2004; Pelli et al., 2007; Pelli \& Tillman, 2008; Strasburger \& Wade, 2015a). For understanding crowding it is paramount to look at its cortical basis, since we know since Flom, Weymouth, \& Kahnemann (1963) that crowding is of cortical origin (as also emphasized by Pelli, 2008).

A question that then arises naturally then is how the cortical equivalent of critical crowding distance varies across the visual field. Klein \& Levi (1987) were the first to consider the cortical distance for position thresholds (in a vernier task), and conclude that it is approximately constant. That conclusion was based on the observation that taking the first derivative of Schwartz's (1980) log mapping using the constancy assumption will result in the well-known inverse-linear cortical magnification function. Conversely, their empirically determined position thresholds, when mapped by an inverse-linear cortical magnification function (with an $E_{2}$ of 0.6 ), turned out mostly constant across a wide rate of eccentricities (cf. their Fig. 5). Later, Duncan and Boynton (2003), after estimating $M$ based on Schwartz's 
(1980) log mapping and applying that to obtain cortical distances (see Section 2.4.2), show that, for scaled vernier tasks and scaled gratings, the cortical equivalents are again mostly constant (above $1.5^{\circ}$ eccentricity; 2003, Fig. 4). Motter \& Simoni, 2007; Pelli, 2008; Mareschal, Morgan, \& Solomon, 2010 $)^{11}$.

Elegant as it seems, however, it will be shown here that the constancy assumption is most likely incorrect (and only true at sufficiently large eccentricities). If stated as a general rule, it rests on the same equating of linearity and proportionality $-i$. e. the omission of the constant term - that gave rise to those cortical location functions that miss the retinotopic center (discussed in Section 2.3). Based on the properties of the cortical location function derived in Section 2., it will turn out that the critical cortical crowding distance (CCCD) increases within the fovea (where reading mostly takes place) and reaches an asymptote beyond perhaps $5^{\circ}$ eccentricity, consistent with a constancy at sufficient eccentricity. Accordingly, Pelli (2008) warns against extrapolating the constancy toward the retinotopic center. Remarkably (and to my pleasant surprise), I found out only after I had completed the derivations, that the analytic equation exposed below nicely agrees with the data presented by Motter \& Simoni (2007, Fig. 7). In that figure, reproduced here in Figure 8B, only the more peripheral data show the presumed constancy.

Let us turn to the equations. Bouma (1970) stated what is now known as Bouma's law for crowding:

$\delta_{\text {space }}=b E$,

where $\delta_{\text {space }}$ is the free space between the patterns at the critical distance ${ }^{12}$ and $b$ is a proportionality factor. Bouma (1970) proposed an approximate value of $b=0.5=50 \%$, which is now widely cited, but he also mentioned that other proportionality factors might work equally well. Indeed, Pelli et al. (2004) have shown that $b$ can take quite different values, depending on the exact visual task. Yet even though this factor varies quite a bit, the implied linearity of eq. (27) holds up in most all the reviewed cases. Bouma specified the distance between target and flankers as free space (the significance of that will become apparent below), and the law is thus best stated as saying that free space for critical spacing is proportional to eccentricity, with the proportionality factor taking some value around $50 \%$, depending on the task (Strasburger, 2020).

Today it has become customary to state flanker distance not as free space but as measured from the respective centers of the target and a flanker. The critical spacing then remains largely constant across sizes (Tripathy \& Cavanagh, 2002, and others). To restate Bouma's rule for the center-to-center distance, $\delta$, let the target pattern have the size $S$ in the radial direction (i.e., width in the horizontal), so that $\delta=S+\delta_{\text {space }}$. Eq. (27) then becomes

$\delta=b E+S$.

This equation no longer represents a proportionality yet is still linear in $E$. Importantly, however, going from Bouma's equation (eq. 27) to that in eq. (28) reflects adding the constant term in the argument that we talked about in the preceding sections, and formally

\footnotetext{
${ }^{11}$ Curiously, these papers do not cite Fischer, 1973, or Schwartz, 1977, who were both earlier in showing the log mapping.

12 To cite from the paper, "an open distance of roughly $0.5 \varphi^{\circ}$ is required for complete isolation" (Bouma, 1970, p. 177, legend to Fig. 2)
} 
that equation (28) is analogous to M-scaling as in eq. (2). Analogously to Levi and Klein's $E_{2}$ we thus introduce a parameter $\hat{E}_{2}$ for crowding where the foveal value of critical distance doubles. Denoting the foveal value of critical distance by $\delta_{0}$, we get, from eq. (28):

$\delta=\delta_{0}\left(E / \hat{E}_{2}+1\right)$.

(29)

Obviously, that equation is analogous to eq. (1) and (2) that we started out with; it describes how critical distance in crowding is linearly dependent on, but is not proportional to, eccentricity in the visual field. In this respect it thus behaves like acuity and many other spatial visual performance measures, just with a different slope.

With the equations derived in the preceding sections, we can now derive the critical crowding distance in the cortical map, i.e. the cortical representation of critical distance in the visual field. Let us denote that distance by $\kappa$ (kappa). By definition, it is the difference between the map locations for the target and a flanker at the critical distance in the crowding task: $\kappa=d_{f}-d_{t}$. The two locations are in turn obtained from the mapping function, which is given by inverting eq. (6) above:

$d=M_{0} E_{2} \ln \left(1+E / E_{2}\right)$, (with $\left.E \geq 0\right)$.

As before, $d$ is the distance of the location in the cortical map from the retinotopic center. So, critical distance $\kappa$ for crowding in the retinotopic map is the difference of the two $d$ values, $\kappa=d_{f}-d_{t}$ :

$$
\kappa=M_{0} E_{2} \ln \left(1+E_{f} / E_{2}\right)-M_{0} E_{2} \ln \left(1+E_{t} / E_{2}\right)=M_{0} E_{2} \ln \frac{\left(1+\left(E_{t}+\delta_{0}\left(E_{t} / \hat{E}_{2}+1\right)\right) / E_{2}\right)}{\left(1+E_{t} / E_{2}\right)}
$$

(by eq. 30 and 29), where $E_{t}$ and $E_{f}$ are the eccentricities for target and flanker, respectively. After simplifying and setting target eccentricity $E_{t}=E$ for generality, this becomes

$$
\kappa=M_{0} E_{2} \ln \left(1+\frac{\delta_{0}}{E_{2}} \frac{\left(1+E / \hat{E}_{2}\right)}{\left(1+E / E_{2}\right)}\right) \text {. }
$$

Note that we stated that equation previously (Strasburger \& Malania, 2013, eq. 13, and Strasburger et al., 2011, eq. 28), but, alas, incorrectly: a factor was missing there.

To explore this function, its graph is shown in Figure $8 \mathrm{~A}$ and we look at two special cases. In the retinotopic center, equation (32) predicts a critical distance $\kappa_{0}$ in the cortical map of

$$
\kappa_{0}=M_{0} E_{2} \ln \left(1+\frac{\delta_{0}}{E_{2}}\right) \text {. }
$$


With increasing eccentricity, $\kappa$ departs from that foveal value and increases (provided $E_{2}>$ $\hat{E}_{2}$ ), depending on the ratio $E_{2} / \hat{E}_{2}$. Numerator and denominator are the $E_{2}$ values for the location function and the crowding function, respectively (eq. 1 vs. eq. 29). They are generally different, so that their ratio is not unity.

With sufficiently large eccentricity, the equation converges to

$$
\lim _{E \rightarrow \infty} \kappa=M_{0} E_{2} \ln \left(1+\frac{\delta_{0}}{\hat{E}_{2}}\right) .
$$

The latter expression is identical to that for the foveal value in eq. (33) except that $E_{2}$ is now replaced by the corresponding value $\hat{E}_{2}$ for crowding.
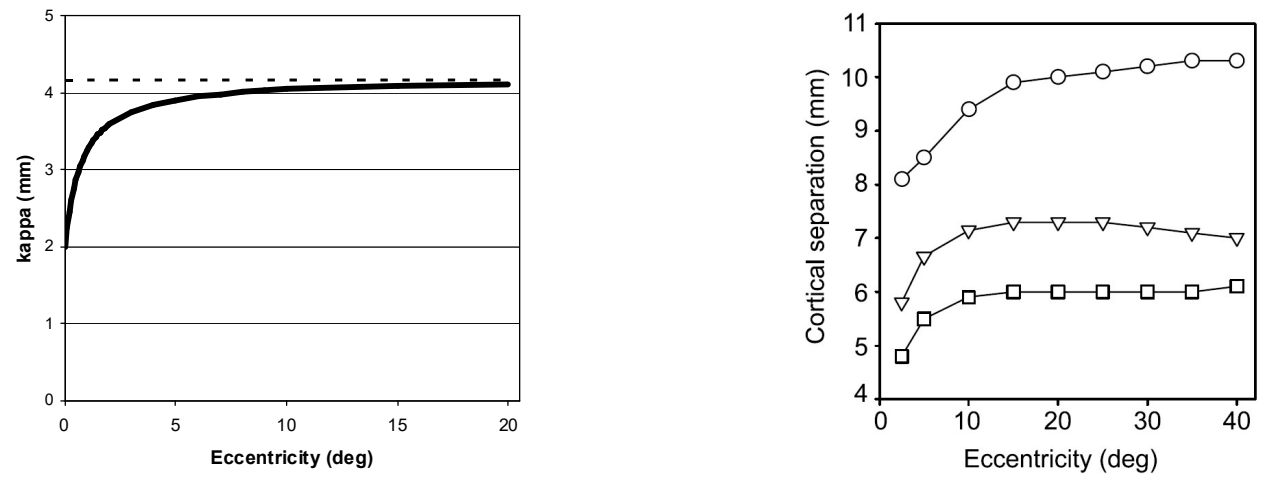

Figure 8. (A) Graph of eq. (32) with realistic values for $M_{0}, E_{2}, \hat{E}_{2}$, and $\delta_{0}$. The value of $E_{2}$ for $M^{-1}$ was chosen as $E_{2}=0.8^{\circ}$ from Dow, Snyder, Vautin, \& Bauer, 1981 (as cited in Levi et al., 1985, or Strasburger et al., 2011, Table 4). $M_{0}=29.1 \mathrm{~mm}$ was chosen to give a good fit with this $E_{2}$ in Fig. 2. Foveal critical distance was set to $\delta_{0}=0.1^{\circ}$ from Siderov, Waugh, \& Bedell, 2013, , 2014. An $\hat{E}_{2}=0.36^{\circ}$ would obtain with this $\delta_{0}$ and the value of $\delta_{4^{\circ}}=1.2^{\circ}$ in Strasburger et al., 1991; it also serves as an example for being a clearly different value than $E_{2}$ for the cortical magnification factor, to see the influence of the $E_{2} / \hat{E}_{2}$ ratio on the graph. Cortical critical distance $\kappa$ starts from the value given in eq. (33) (around $2 \mathrm{~mm}$ ) and converges to the value in eq. (34). (B) Data for the cortical critical distance from Motter \& Simoni (2007, Fig. 7).

Importantly, note that kappa varies substantially around the center, by around two-fold between the center and $5^{\circ}$ eccentricity with realistic values of $E_{2}$ and $\hat{E}_{2}$. This, as said above, is at odds with the conjecture that the cortical critical crowding distance is a constant (Motter \& Simoni, 2007; Pelli, 2008; Mareschal et al., 2010). Pelli (2008) presented a mathematical derivation for the constancy, very similar to the one presented above - based on Bouma's law and Schwartz' (1980) logarithmic mapping function. The discrepancy arises from the assumptions: Pelli used Bouma's law as proportionality, i.e., in its simplified form stated in eq. (27) (its graph passing through the origin). The simplification was done on the grounds that the error is small outside the retinotopic center and plays little role (and the paper appropriately warns that additional provisions must be made at small eccentricities). Schwartz' (1980) mapping function was consequently also used in its simplified form (also leaving out the constant term), for the same reason. With these simplifications the critical distance in the cortex indeed turns out as simply being a constant. 
As should be expected, at sufficiently high eccentricities $\kappa$ is close to constant in the derivations given above (Figure 8). These equations (eq. 32-34) can thus be seen as a generalization of Pelli's result that now also covers the (obviously important) case of central vision.

That said, an interesting (though unlikely) special case of eq. (32) is the one in which $E_{2}$ and $\hat{E}_{2}$ are equal. $\kappa$ is then a constant, as Pelli (2008) predicted. Its value in that case would be simply given by

$$
\kappa=M_{0} E_{2} \ln \left(1+\frac{\delta_{0}}{E_{2}}\right) \text {, for } E_{2}=\hat{E}_{2} .
$$

On a different note, equations (32)-(35) have $M_{0}$ as a scaling factor and, as said before, $M_{0}$ is notoriously difficult to determine empirically. However, $M_{0}$ can be replaced, as shown above. From eq. (17) we know that

$$
M_{0} E_{2}=\frac{d_{r e f}}{\beta},
$$

which, by the definition of $\beta$, takes a particularly simple form when $d_{2}$ (the cortical equivalent of $E_{2}$ ) is chosen as the reference:

$$
M_{0} E_{2}=\frac{d_{2}}{\ln 2}
$$

(which is the same as eq. 8a). We can then rewrite the equation for the cortical crowding critical distance (eq. 32) as

$$
\kappa=\frac{d_{2}}{\ln 2} \ln \left(1+\frac{\delta_{0}}{E_{2}} \frac{\left(1+E / \hat{E}_{2}\right)}{\left(1+E / E_{2}\right)}\right) \text {. }
$$

Similarly, the two special cases eq. (33) and (34) become

$$
\kappa_{0}=\frac{d_{2}}{\ln 2} \ln \left(1+\frac{\delta_{0}}{E_{2}}\right)
$$

and

$$
\lim _{E \rightarrow \infty} \kappa=\frac{d_{2}}{\ln 2} \ln \left(1+\frac{\delta_{0}}{\hat{E}_{2}}\right) .
$$

Values for $d_{2}$ derived from the literature by eq. (37) that could be plugged into eq. (39) and (40) were provided in Table 2 above. These two equations, for the retinotopic center and eccentricities above around $5^{\circ}$, respectively, could lend themselves for determining critical crowding distance in the cortex.

In summary for the cortical crowding distance, the linear eccentricity laws in psychophysics for cortical magnification and for critical crowding distance - both well established together with Fischer's (1973) or Schwartz's (1977; 1980) equally well-established 
logarithmic mapping rule, predict a highly systematic behavior of crowding's critical distance in the cortical map. Given the very similar mappings in areas V2, V3, V4 (Larsson \& Heeger, 2006; Schira et al., 2009), that relationship can be expected to hold in those areas as well (see Figure $8 \mathrm{~A}$ for a graph). Since the cortical location function is well established and the equations for crowding follow mathematically, they should work well with suitable $E_{2}$ values inserted. Thus, direct confirmations of their behavior would cross-validate mapping models and might shed light on the mechanisms underlying crowding.

\section{Outlook}

Where does this leave us? The early cortical visual areas are very regularly organized. And, as apparent from the $\mathrm{fMRI}$ literature reviewed above and also earlier literature, the spatial maps of early visual areas appear to be pretty similar. Yet variations of visual performance across the visual field differ widely between visual tasks, as highlighted, e.g., by their respective, widely differing $E_{2}$ values. $E_{2}$ estimates for cortical magnification, on the other hand, appear to be quite similar. The puzzle of how different spatial scalings in psychophysics emerge on a uniform cortical architecture is still unresolved. Certainly, however, there can be only one valid location function on any radius; so the equivalence between psychophysical $E_{2}$ and the cortical location function in the preceding equations can only hold for a single $E_{2}$. That value is probably the one pertaining to certain low-level tasks, and likely to those tasks that are somehow connected to stimulus size. In contrast, $\hat{E}_{2}$ for critical crowding distance is an example for a psychophysical descriptor that is not linked to stimulus size (Pelli et al., 2004); it rather reflects location differences, as discussed in Section 4. The underlying cortical architecture that brings about different psychophysical $E_{2}$ values (like $\hat{E}_{2}$ ) could be neural wiring differences, within or between early visual areas.

To go further, one of the basic messages of the cortical-magnification literature is the realization that by $M$-scaling stimulus sizes some, but not all, performance variations are equalized across the visual field (Virsu et al., 1987; Strasburger et al., 2011, Section 2.5). In parameter space, these other variables would be said to be orthogonal to target size. For pattern recognition, pattern contrast is such a variable (Strasburger, Rentschler, \& Harvey, 1994; Strasburger \& Rentschler, 1996). Temporal resolution is another example (Poggel, Calmanti, Treutwein, \& Strasburger, 2012). Again, differing patterns of connectivity between retinal cell types, visual areas, and along different processing streams might underlie these performance differences. The aim of the present paper is just to point out that a common spatial location function underlies the early cortical architecture that can be described by a unified equation. This equation includes the fovea and the retinotopic center, and has parameters that are common in psychophysics and physiology.

\section{Acknowledgements}

I thank Dany d'Souza for the original question, Barry Lee for critical comments on the manuscript and meticulous language corrections, Zhaoping Li and Josh Solomon for critical reading, and Zhaoping Li for a thorough check of the mathematical derivations, and an anonymous reviewer for pointing out Klein \& Levi (1987).

\section{References}

Aubert, H. R., \& Foerster, C. F. R. (1857). Beiträge zur Kenntniss des indirecten Sehens. (I). Untersuchungen über den Raumsinn der Retina. Archiv für Ophthalmologie, 3, 1-37. 
Bouma, H. (1970). Interaction effects in parafoveal letter recognition. Nature, 226, 177-178.

Cowey, A., \& Rolls, E. T. (1974). Human cortical magnification factor and its relation to visual acuity. Experimental Brain Research, 21, 447-454.

D'Souza, D. V., Auer, T., Strasburger, H., Frahm, J., \& Lee, B. B. (2016). Dependence of chromatic response in $\mathrm{V} 1$ on visual field eccentricity and spatial frequency: an fMRI study. Journal of the Optical Society of America A, 33(3), A53-A64.

Daniel, P. M., \& Whitteridge, D. (1961). The representation of the visual field on the cerebral cortex in monkeys. Journal of Physiology, 159, 203-221.

Dow, B. M., Snyder, R. G., Vautin, R. G., \& Bauer, R. (1981). Magnification factor and receptive field size in foveal striate cortex of the monkey. Experimental Brain Research, 44, 213-228.

Duncan, R. O., \& Boynton, G. M. (2003). Cortical magnification within human primary visual cortex. Correlates with acuity thresholds. Neuron, 38, 659-671.

Engel, S. A., Glover, G. H., \& Wandell, B. A. (1997). Retinotopic organization in human visual cortex and the spatial precision of functional MRI. Cerebral Cortex, 7, 181-192.

Fischer, B. (1973). Overlap of receptive field centers and representation of the visual field in the cat's optic tract. Vision Research, 13, 2113-2120.

Flom, M. C., Weymouth, F. W., \& Kahnemann, D. (1963). Visual resolution and contour interaction. J. Opt. Soc. Am. 53, 1026-1032.

Greenwood, J., Danter, J., \& Finnie, R. (2017). Cortical distance determines the perceptual outcomes of crowding. Journal of Vision, 17, 398.

Harvey, L. O., Jr., \& Pöppel, E. (1972). Contrast sensitivity of the human retina. American Journal of Optometry and Archives of the American Academy of Optometry, 49, 748-753.

Horton, J. C., \& Hoyt, W. F. (1991). The representation of the visual field in human striate cortex. A revision of the classic Holmes map. Archives of Ophthalmology, 109(6), 816-824.

Jurin, J. (1738). An essay on distinct and indistinct vision. In A compleat system of opticks in four books, viz. a popular, a mathematical a mechanical, and a philosophical treatise (pp. 115-171). Cambridge: Published by the author.

Klein, S. A., \& Levi, D. M. (1987). Position sense of the peripheral retina. Journal of the Optical Society of America A, 4(8), 1543-1553.

Larsson, J., \& Heeger, D. J. (2006). Two retinotopic visual areas in human lateral occipital cortex. The Journal of Neuroscience, 26(51), 13128-13142.

Levi, D. M., Klein, S. A., \& Aitsebaomo, A. P. (1984). Detection and discrimination of the direction of motion in central and peripheral vision of normal and amblyopic observers. Vision Research, 24, 789-800.

Levi, D. M., Klein, S. A., \& Aitsebaomo, A. P. (1985). Vernier acuity, crowding and cortical magnification. Vision Research, 25, 963-977.

Mareschal, I., Morgan, M. J., \& Solomon, J. A. (2010). Cortical distance determines whether flankers cause crowding or the tilt illusion. Journal of Vision, 10(8), 13:11-14.

Motter, B. C., \& Simoni, D. A. (2007). The roles of cortical image separation and size in active visual search performance. Journal of Vision, 7(2:6), 1-15.

Nandy, A. S., \& Tjan, B. S. (2012). Saccade-confounded image statistics explain visual crowding. Nature Neuroscience, 15(3), 463-471.

Oehler, R. (1985). Spatial interactions in the rhesus monkey retina: a behavioural study using the Westheimer paradigm. Experimental Brain Research, 59, 217-225.

Osterberg, G. (1935). Topography of the layer of rods and cones in the human retina. Acta Ophthalmologica. Supplement, 6-10, 11-96.

Pelli, D. G. (2008). Crowding: a cortical constraint on object recognition. Current Opinion in Neurobiology, 18, 445-451.

Pelli, D. G., Palomares, M., \& Majaj, N. J. (2004). Crowding is unlike ordinary masking: Distinguishing feature integration from detection. Journal of Vision, 4(12), 1136-1169.

Pelli, D. G., \& Tillman, K. A. (2008). The uncrowded window of object recognition. Nature Neuroscience, 11(10), 1129-1135 (plus online supplement). 
Pelli, D. G., Tillman, K. A., Freeman, J., Su, M., Berger, T. D., \& Majaj, N. J. (2007). Crowding and eccentricity determine reading rate. Journal of Vision, $7(2)$.

Poggel, D. A., Calmanti, C., Treutwein, B., \& Strasburger, H. (2012). The Tölz Temporal Topography Study: Mapping the visual field across the life span. Part II: Cognitive factors shaping visual field maps. Attention, Perception \& Psychophysics, 74, 1133-1144.

Pöppel, E., \& Harvey, L. O., Jr. (1973). Light-difference threshold and subjective brightness in the periphery of the visual field. Psychologische Forschung, 36, 145-161.

Rovamo, J., \& Virsu, V. (1979). An estimation and application of the human cortical magnification factor. Experimental Brain Research, 37, 495-510.

Rovamo, J., Virsu, V., \& Näsänen, R. (1978). Cortical magnification factor predicts the photopic contrast sensitivity of peripheral vision. Nature, 271, 54-56.

Schira, M. M., Tyler, C. W., Breakspear, M., \& Spehar, B. (2009). The foveal confluence in human visual cortex. The Journal of Neuroscience, 29 (July 15), 9050 -9058.

Schira, M. M., Tyler, C. W., Spehar, B., \& Breakspear, M. (2010). Modeling magnification and anisotropy in the primate foveal confluence. PLoS Computational Biology, 6(1), e1000651.

Schira, M. M., Wade, A. R., \& Tyler, C. W. (2007). Two-dimensional mapping of the central and parafoveal visual field to human visual cortex. Journal of Neurophysiology [Epub ahead of print], 97(6), 4284-4295.

Schwartz, E. L. (1977). Spatial mapping in the primate sensory projection: Analytic structure and relevance to perception. Biological Cybernetics, 25, 181-194.

Schwartz, E. L. (1980). Computational anatomy and functional architecture of striate cortex: A spatial mapping approach to perceptual coding. Vision Research, 20, 645-669.

Siderov, J., Waugh, S. J., \& Bedell, H. E. (2013). Foveal contour interaction for low contrast acuity targets. Vision Research, 77, 10-13.

Siderov, J., Waugh, S. J., \& Bedell, H. E. (2014). Foveal contour interaction on the edge: response to 'letter-to-the-editor' by Drs. Coates and Levi. Vision Research, 96, 145-148.

Slotnick, S. D., Klein, S. A., Carney, T., \& Sutter, E. E. (2001). Electrophysiological estimate of human cortical magnification. Clinical Neurophysiology, 112(7), 1349-1356.

Strasburger, H. (2020). Seven myths on crowding and peripheral vision (Preprint). PeerJ Preprints, 6:e27250.

Strasburger, H., Harvey, L. O. J., \& Rentschler, I. (1991). Contrast thresholds for identification of numeric characters in direct and eccentric view. Perception \& Psychophysics, 49, 495-508.

Strasburger, H., \& Malania, M. (2013). Source confusion is a major cause of crowding. Journal of Vision, 13(1), 1-20.

Strasburger, H., \& Rentschler, I. (1996). Contrast-dependent dissociation of visual recognition and detection field. European Journal of Neuroscience, 8(8), 1787-1791.

Strasburger, H., Rentschler, I., \& Harvey, L. O., Jr. (1994). Cortical magnification theory fails to predict visual recognition. European Journal of Neuroscience, 6, 1583-1588.

Strasburger, H., Rentschler, I., \& Jüttner, M. (2011). Peripheral vision and pattern recognition: a review. Journal of Vision, 11(5), 1-82.

Strasburger, H., \& Wade, N. J. (2015a). James Jurin (1684-1750): A pioneer of crowding research? Journal of Vision, 15(1:9), 1-7.

Strasburger, H., \& Wade, N. J. (2015b). James Jurin (1684-1750): A pioneer of crowding research? Poster presented at the ECVP 2015, Liverpool. F1000Research, 4:675.

Tolhurst, D. J., \& Ling, L. (1988). Magnification factors and the organization of the human striate cortex. Human Neurobiology, 6, 247-254.

Trevarthen, C. B. (1968). Two Mechanisms of Vision in Primates. Psychologische Forschung, 31, 299-337.

Tripathy, S. P., \& Cavanagh, P. (2002). The extent of crowding in peripheral vision does not scale with target size. Vision Research, 42, 2357-2369. 
Van Essen, D. C., Newsome, W. T., \& Maunsell, J. H. R. (1984). The visual field representation in striate cortex of the macaque monkey: Asymmetries, anisotropies, and individual variability. Vision Research, 24(5), 429-448.

Virsu, V., \& Hari, R. (1996). Cortical magnification, scale invariance and visual ecology. Vision Research, 36(18), 2971-2977.

Virsu, V., Näsänen, R., \& Osmoviita, K. (1987). Cortical magnification and peripheral vision. Journal of the Optical Society of America A, 4, 1568-1578.

Virsu, V., \& Rovamo, J. (1979). Visual resolution, contrast sensitivity and the cortical magnification factor. Experimental Brain Research, 37, 475-494.

Wade, N. J. (1998). A Natural History of Vision (A Bradford Book). Cambridge: MIT Press.

Watson, A. B. (1987). Estimation of local spatial scale. Journal of the Optical Society of America A, 4(8), 1579-1582.

Wertheim, T. (1894). Über die indirekte Sehschärfe. Zeitschrift für Psycholologie \& Physiologie der Sinnesorgane, 7, 172-187.

Westheimer, G. (1982). The spatial grain of the perifoveal visual field. Vision Research, 22, 157-162.

Weymouth, F. W. (1958). Visual sensory units and the minimal angle of resolution. American Journal of Ophthalmology, 46, 102-113. 